\title{
Local scale-invariance in disordered systems
}

\author{
Malte Henkel ${ }^{1}$ and Michel Pleimling ${ }^{2}$ \\ ${ }^{1}$ Laboratoire de Physique des Matériaux, Université Henri Poincaré Nancy I, B.P. \\ 239, F - 54506 Vandœuvre-lès-Nancy Cedex, France \\ ${ }^{2}$ Department of Physics, Virginia Polytechnic Institute and State University, \\ Blacksburg, Virginia 24061-0435, USA
}

Summary. Dynamical scaling and ageing in disordered systems far from equilibrium is reviewed. Particular attention is devoted to the question to what extent a recently introduced generalization of dynamical scaling to local scale-invariance can describe data for either non-glassy systems quenched to below $T_{c}$ or else for spin glasses at criticality. The dependence of the scaling behaviour on the distribution of the random couplings is discussed. It is shown that finite-time corrections to scaling can become quite sizable in these systems. Numerically determined ageing quantities are confronted with available experimental results.

\section{Introduction}

Understanding cooperative phenomena far from equilibrium poses one of the most challenging research problems of present-day many-body physics. At the same time, the practical handling of many of these materials has been pushed to great sophistication, and a lot of practical knowledge about them exists since prehistoric times. Paradigmatic examples of such system are glasses. In many cases, they are made by rapidly cooling ('quenching') a molten liquid to below some characteristic temperature-threshold. If this cooling happens rapidly enough, normal crystallization no longer takes place and the material remains in some non-equilibrium state. These non-equilibrium states may at first and even second sight look very stationary - everyone has probably seen in archaeological museums intact specimens of Roman glass or even older tools from the Paleolithic or old-stone-age - after all, obsidian or fire-stone is a quenched volcanic melt. But since the material is not at equilibrium, at least in principle it is possible (and it does happen very often in practice) that over time the properties of the material change - in other words, the material ages. ${ }^{1}$ The properties of such non-equilibrium systems depend on the time - their age since they were brought out of equilibrium and this is colloquially referred to as ageing behaviour.

\footnotetext{
${ }^{1}$ Recall that physical ageing as it is understood here comes from reversible microscopic processes, whereas chemical or biological ageing may come from the action of essentially irreversible (bio-)chemical processes.
} 
Although the first systematic studies of ageing were carried out in the by now classic experiments of Struik [116] in polymeric glasses, it has been realized in recent years that very similar phenomena already occur in non-disordered and nonfrustrated systems which hence are also commonly referred to as ageing. In order to present the essence of the ageing phenomenon, we begin by considering a simple Ising model, made from spin variables $\sigma_{i}= \pm 1$ attached to each site $i$ of a hypercubic lattice and an interaction as given by the classical hamiltonian with the usual nearest-neighbour interactions

$$
\mathcal{H}=-J \sum_{(i, j)} \sigma_{i} \sigma_{j}
$$

where $J>0$ is the exchange integral and the sum extends over pairs of nearest neighbours. In $d>1$ dimensions, this system has a phase-transition at some critical temperature $T_{c}>0$ which separates a disordered phase at high temperatures with a single thermodynamically stable state from an ordered phase at low temperatures with two thermodynamically stable equilibrium states. It is well-established that such simple Ising models may be used to discuss uniaxial magnets, binary alloys or even liquid crystals. The motion of the spins is generated by coupling the model to a thermal bath of temperature $T$. A possible way of realizing this is through the so-called heat-bath dynamics which is defined by the stochastic rule

$$
\sigma_{i}(t+\Delta t)= \pm 1 \quad \text { with probability }\left[1 \pm \tanh \left(h_{i}(t) / T\right)\right] / 2
$$

where $\Delta t$ is the time increment, the local time-dependent field is $h_{i}(t)=\sum_{y(i)} \sigma_{y(i)}(t)$ and $y(i)$ runs over the nearest neighbours of the site $i$. It is well-known that this rule satisfies detailed balance and hence the system evolves towards the equilibrium probability distribution $P_{\text {eq }}=Z^{-1} \exp (-\mathcal{H} / T)$, where $Z$ is the canonical partition function $[118,93,123]$. The system is prepared at some initial temperature $T_{\text {ini }}$ far above the critical temperature $T_{c}>0$. The initial time $t=0$ is defined by coupling the system to the thermal bath at some low temperature $T<T_{c}$ and starting the dynamics. During the simulation, the temperature $T$ is kept fixed and one observes the time-dependence of observables such as correlation functions or susceptibilities. ${ }^{2}$

Qualitatively, the behaviour of the system can be illustrated through the equilibrium free energies at the temperatures $T_{\text {ini }}$ and $T$, see figure 1 . Before the quench, the system is at equilibrium with respect to the initial temperature $T_{\text {ini }} \gg T_{c}$ and sits at the minimum of the free energy, as indicated by the black ball in figure 1a. If one would perturbe this system slightly, it would relax rapidly, i.e. with a finite relaxation time $0<\tau<\infty$, towards this unique equilibrium state. On the other hand, immediately after the quench the system does not yet have had the time to evolve but, with respect to the new equilibrium, its free energy is no longer minimal, see figure 1b. Rather, two new local minima of the free energy appear, which correspond to the two equivalent ordered states of the system. Because of the competition between these two equivalent equilibrium states, the system as a whole cannot relax rapidly to one of them but rather undergoes a slow dynamics, with formally infinite relaxation times. Locally, each spin will be subject to the time-dependent field

\footnotetext{
${ }^{2}$ The chosen dynamics is such that the total average magnetization $M(t)=$ $\langle\sigma(t)\rangle=\sum_{i}\left\langle\sigma_{i}(t)\right\rangle$ remains at its initial value $M(0)=0$. Throughout, we shall always use this initial condition unless explicitly stated otherwise.
} 

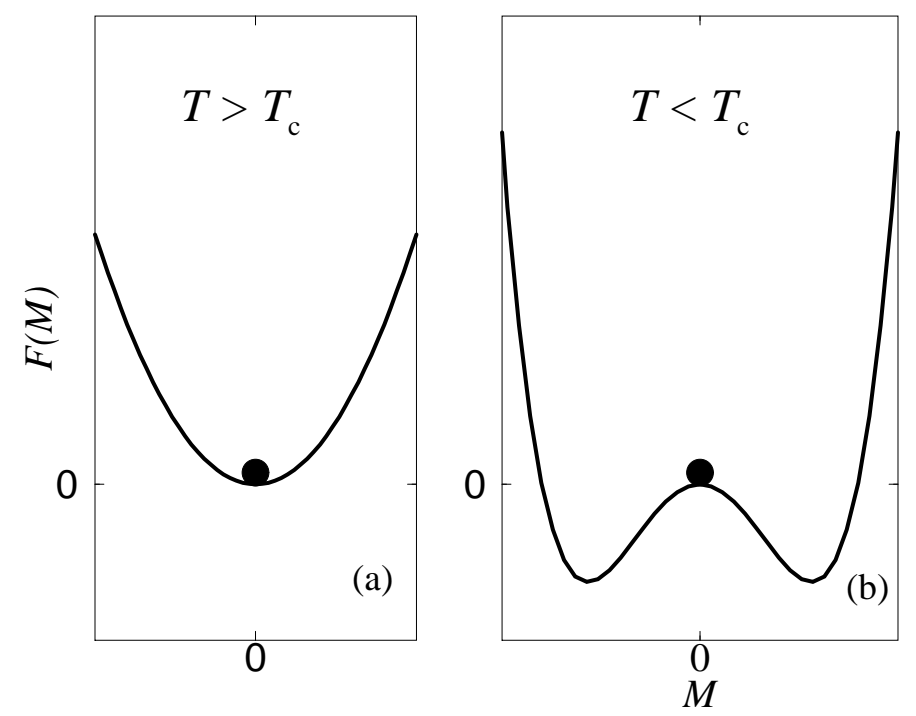

Fig. 1. Free energy $F(M)$ as a function of the magnetization $M$ of a simple ferromagnet at (a) an initial high temperature $T>T_{c}$ before the quench and (b) after the quench to a low temperature $T<T_{c}$.

$h_{i}(t)$ coming from its neighbours and this field will tilt the balance between the two equivalent equilibrium states of figure $1 \mathrm{~b}$ in favour of one or the other. Physically, this means that the system will rapidly decompose into ordered domains and the slow long-time dynamics of this domain growth will be determined by the motion of the domain walls between these ordered domains. This slow (non-exponential) dynamics is the first defining property of ageing systems.

Another aspect of this non-equilibrium dynamics (since in a spatially infinite system none of the equilibrium states will be reached in a finite time) becomes apparent if one considers a quantity like the two-time autocorrelation function of spins at site $i$ at times $t$ and $s$

$$
C(t, s)=\left\langle\sigma_{i}(t) \sigma_{i}(s)\right\rangle
$$

which by spatial translation-invariance is independent of the chosen site $i$. In figure 2a data for $C(t, s)$ plotted over against the time difference $t-s$ are displayed for the three-dimensional Ising model. We see that the data depend on both $t-s$ and $s$, hence time-translation invariance is broken and the system ages. Further, with increasing values of the waiting time $s$, the system becomes 'stiffer' and a plateau close to the equilibrium value $C_{\mathrm{eq}}=M_{\mathrm{eq}}^{2}$ develops when $t-s$ is not too large before the correlations fall off rapidly when $t-s \rightarrow \infty$. Together with the slow dynamics mentioned above, this breaking of time-translation invariance is the second defining property of ageing systems. In principle, this could mean that the details of the dynamics of ageing systems might depend on the entire prehistory of the sample under study, which would make any attempt to formulate a general theory for such systems hopeless. However, a great simplification, due to dynamical scaling, is apparent in figure $2 \mathrm{~b}$ where the same data for $C(t, s)$, when plotted over 

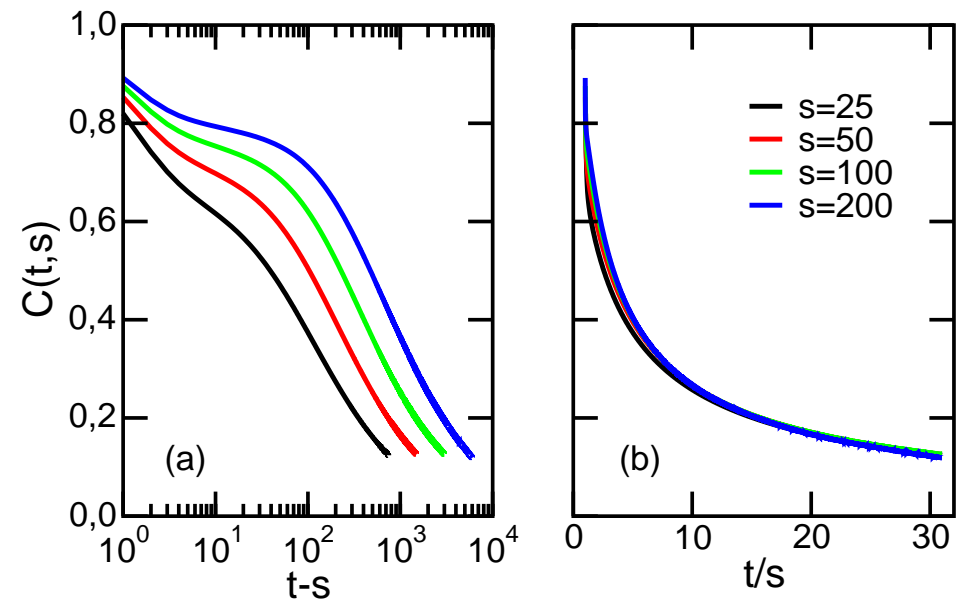

Fig. 2. (a) Ageing and (b) dynamical scaling of the two-time autocorrelation function $C(t, s)$ in the three-dimensional Glauber-Ising model quenched to $T=3<T_{c}$, for several values of the waiting time $s$.

against $t / s$, neatly collapse onto a single curve, if only the time $s$ is large enough and also $t-s$ is not too small. Since in domain coarsening of simple magnets one expects that the linear size of the ordered domains is $L=L(t) \sim t^{1 / z}$ when $t$ is large enough and $z$ is the dynamical exponent [20], the collapse in figure $2 \mathrm{~b}$ means that $C(t, s)=f(L(t) / L(s))$, or in other words $L(t)$ is the only relevant length-scale at time $t$. Dynamic scaling (although more general forms for $L(t)$ than simple power laws are of course possible) is the third essential property of ageing systems.

These three basic properties of ageing systems are also found in glassy systems. An important property of glasses is the presence of frustrations which prevent the relaxation of all local degrees of freedom. In consequence, the free-energy landscape of glasses can be very complex, with many local minima. The classic example for ageing behaviour was observed by Struik [116] in studying the mechanical properties of polymeric glasses which after a quench from the molten phase to low temperatures (i) relax very slowly (typical time-scale of years), (ii) show clear evidence of the breaking of time-translation invariance and furthermore, (iii) the experimental data for the time-dependent creep curves of the mechanical response can all be mapped onto a single master curve. Remarkably, that master curve turned out to be the same for materials as different as polymers such as PVC or PMMA, sugar or even metals like lead ! Evidently, there are universal scaling functions in ageing which exactly because of their universality one may hope to be able to understand theoretically. Returning to simple magnets, that universality in the kinetics of coarsening (with a non-conserved order-parameter) after a quench to $T<T_{c}$ is captured through the celebrated Allen-Cahn equation [3] which states that the velocity $v$ of the domain walls which separate the ordered domains is related to the curvature $K$ in $d$ dimensions via the purely geometric relationship $v=(d-1) K$, quite independently of any details of the interactions of the spins.

The microscopic evolution which underlies the above statements about the properties of macroscopic observables is illustrated in figure 3. In the first column, snap- 

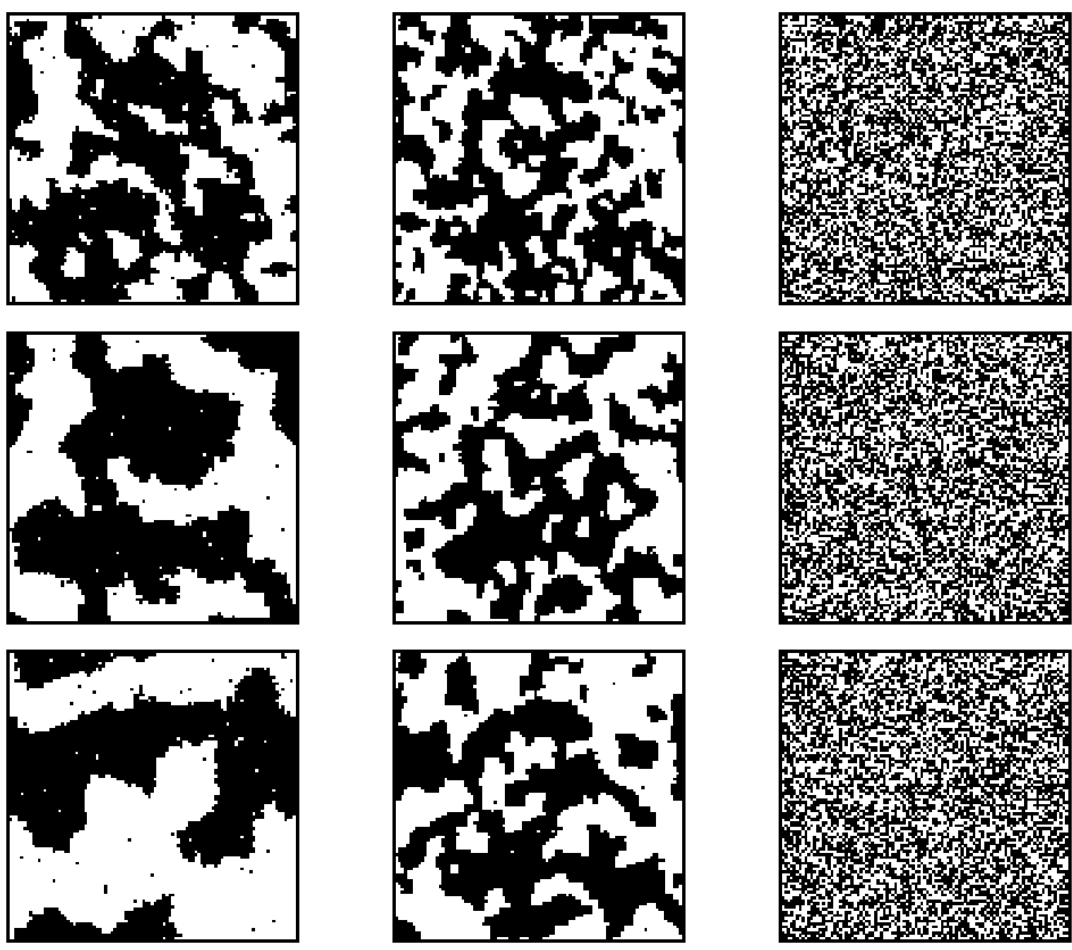

Fig. 3. Snapshots illustrating the growth of clusters in three variants of the kinetic Ising model, at times $t=25,100$ and 225 after the quench, from top to bottom. The left column shows the two-dimensional Glauber-Ising model without disorder quenched to $T=1.5<T_{c}$ and the middle column shows the random bond Ising model in two dimensions with $\varepsilon=2$ and $T=0.7<T_{c}$. The right column shows a section of the three-dimensional Ising spin glass with binary disorder at $T=0.8<$ $T_{c}$.

shots of configurations of the two-dimensional Glauber-Ising model without disorder are shown for three different times after the quench from a fully disordered initial configuration into the ordered phase. Clearly, ordered domains form very rapidly and continue to grow up to the system size. In order to appreciate the effects of disorder, we next consider a random-bond Ising model defined by the classical hamiltonian

$$
\mathcal{H}_{\mathrm{dis}}=-\sum_{(i, j)} J_{i, j} \sigma_{i} \sigma_{j}
$$

where the $J_{i, j}$ are random variables uniformly distributed over the interval [ 1 $\varepsilon / 2,1+\varepsilon / 2]$ with $0<\varepsilon \leq 2$. Snapshots from this two-dimensional model are shown for $\varepsilon=2$ and $T=0.7<T_{c}$ in the middle column of figure 3. Again, detectable ordered domains from rapidly but with respect to the non-disordered Glauber-Ising model, although the clusters look broadly similar, one sees that the growth of the clusters proceeds much more slowly. Finally, we consider an Ising spin glass, which can again be defined by the hamiltonian (4) but now the random couplings $J_{i, j}= \pm 1$ 
can take both positive and negative values with equal probabilites. The right column in figure 3 illustrates the evolution of the three-dimensional Ising spin glass with binary disorder $\left(J_{i, j}= \pm 1\right.$ with equal probability $)$ at the temperature $T=0.8<T_{c}$. It is clear that the presence of both ferromagnetic and antiferromagnetic interactions no longer permits the formation of ordered clusters and with respect to the two other cases, the evolution appears considerably slower. We shall formalize this later on when we discuss the growth laws of the time-dependent characteristic length.

Universality as seen in experiments [116] or manifest in the Allen-Cahn equation may signal the presence of deeper dynamical symmetries. In this review, we shall describe the available evidence which points to the possibility that the wellestablished dynamical scaling found in ageing systems may be included into a larger group of local scale-transformations. As a necessary preparation we shall begin by briefly recalling the main features of dynamical scaling. The topic has been under intensive study, see $[20,29,34,49,33,82,25,46,64,67]$ for reviews.

It is useful to discuss the properties of the coarse-grained order-parameter, denoted by $\phi(t, \boldsymbol{r})$ at time $t$ at the location $\boldsymbol{r}$. Most of our discussion is for the case that the order-parameter is non-conserved by the dynamics and that the initial state is totally disordered with $\langle\phi(0, \boldsymbol{r})\rangle=0$, unless explicitly stated otherwise. It has become common to study ageing behaviour through the two-time autocorrelation and (linear) autoresponse functions

$$
\begin{aligned}
& C(t, s)=\langle\phi(t, \boldsymbol{r}) \phi(s, \boldsymbol{r})\rangle \\
& R(t, s)=\left.\frac{\delta\langle\phi(t, \boldsymbol{r})\rangle}{\delta h(s, \boldsymbol{r})}\right|_{h=0} \sim s^{-b} f_{C}(t / s) \\
& \sim s^{-1-a} f_{R}(t / s)
\end{aligned}
$$

where $h(s, \boldsymbol{r})$ is the magnetic field conjugate to $\phi$ at time $s$ and location $\boldsymbol{r}$. The scaling behaviour is expected to apply in the so-called ageing regime where

$$
t, s \gg t_{\text {micro }} \text { and } t-s \gg t_{\text {micro }}
$$

where $t_{\text {micro }}$ is a microscopic time-scale. We emphasize the importance of the third condition for the validity of dynamical scaling. This makes the above qualitative observations drawn from figure 2 more precise. In particular, we see in figure 2 that for finite values of $s$ scaling is not perfect: indeed corrections to dynamical scaling are detectable when $t / s \approx 1$.

The distance of such systems from a global equilibrium state can be measured through the fluctuation-dissipation ratio, defined as [35]

$$
X(t, s):=T R(t, s)\left(\frac{\partial C(t, s)}{\partial s}\right)^{-1} .
$$

At equilibrium, $X(t, s)=1$ from the fluctuation-dissipation theorem. One often considers the limit fluctuation-dissipation ratio ${ }^{3}$

$$
X_{\infty}:=\lim _{s \rightarrow \infty}\left(\lim _{t \rightarrow \infty} X(t, s)\right)=\lim _{y \rightarrow \infty}\left(\left.\lim _{t, s \rightarrow \infty} X(t, s)\right|_{y=t / s}\right) .
$$

For quenches to below $T_{c}$, one usually has $X_{\infty}=0$ but for critical quenches onto $T=T_{c}$, it has been proposed by Godrèche and Luck that $X_{\infty}$ should be a universal number [48], since it can be written as a ratio of two scaling amplitudes.

\footnotetext{
${ }^{3}$ The order of the limits is crucial, since $\lim _{t \rightarrow \infty}\left(\lim _{s \rightarrow \infty} X(t, s)\right)=1$.
} 
The universality of $X_{\infty}$ has been thoroughly confirmed for systems relaxing towards equilibrium steady-states, and can be theoretically validated in a field-theoretical setting, see [33, 25] for recent reviews. On the other hand, we mention here that quite similar scaling behaviour is also found for systems containing irreversible chemical reactions and hence relaxing towards non-equilibrium steady-states. In that case, the definitions of $X(t, s)$ and of $X_{\infty}$ have to be reconsidered, see [67] for a recent review.

Furthermore, in writing eqs. $(5,6)$ it was tacitly assumed that the scaling derives from the algebraic time-dependence of a single characteristic length-scale growing algebraically with time $L(t) \sim t^{1 / z}$ which measures the linear size of correlated or ordered clusters and where $z$ is the dynamic exponent. That is indeed the case for simple magnets whose kinetics may be described in terms of a simple Ising model, e.g. with Glauber-type dynamics, where it can even be shown that $z=2[20,21]$. Then the above forms $(5,6)$ define the non-equilibrium exponents $a$ and $b$ and the scaling functions $f_{C}(y)$ and $f_{R}(y)$. For large arguments $y \rightarrow \infty$, one generically expects

$$
f_{C}(y) \sim y^{-\lambda_{C} / z}, f_{R}(y) \sim y^{-\lambda_{R} / z}
$$

where $\lambda_{C}$ and $\lambda_{R}$, respectively, are known as autocorrelation [44, 73] and autoresponse exponents [102].

While in non-disordered magnets with short-ranged initial conditions one usually has $\lambda_{C}=\lambda_{R}$, this is not necessarily so if either of these conditions is relaxed. From a field-theoretical point of view it is known that for a non-conserved order-parameter the calculation of $\lambda_{C, R}$ requires an independent renormalization and hence one cannot expect to find a scaling relation between these and equilibrium exponents (including $z$ ) [76]. On the other hand, the values of the exponents $a$ and $b$ are known. For quenches to $T=T_{c}$, the relevant length-scale is set by the time-dependent correlation length $L(t) \sim \xi(t) \sim t^{1 / z}$ and this leads to $a=b=(d-2+\eta) / z$, where $\eta$ is a standard equilibrium exponent. For quenches into the ordered phase $T<T_{c}$, one usually observes simple scaling of $C(t, s)=f_{C}(t / s)$, hence $b=0 .{ }^{4}$ The value of $a$ depends on whether the equilibrium correlator is short- or long-ranged, respec-

Table 1. Values of the non-equilibrium exponents $a, b$ and $z$ for non-conserved ferromagnets with $T_{c}>0$ and a vanishing initial magnetization. The non-trivial critical-point value $z_{c}$ is model-dependent.

\begin{tabular}{|l|lll|l|}
\hline \hline & $a$ & $b$ & $z$ & Class \\
\hline \hline$T=T_{c}$ & $(d-2+\eta) / z$ & $(d-2+\eta) / z$ & $z_{c}$ & $\mathrm{~L}$ \\
\hline$T<T_{c}$ & $(d-2+\eta) / z$ & 0 & 2 & $\mathrm{~L}$ \\
& $1 / z$ & 0 & 2 & $\mathrm{~S}$ \\
\hline \hline
\end{tabular}

\footnotetext{
${ }^{4}$ This needs no longer be the case when the ageing close to a free surface is considered [10].
} 
tively. These may be referred to as classes $\mathrm{S}$ and L, respectively, and one has, see e.g. $[29,56,60]$

$$
C_{\mathrm{eq}}(\boldsymbol{r}) \sim\left\{\begin{array} { l } 
{ e ^ { - | \boldsymbol { r } | / \xi } } \\
{ | \boldsymbol { r } | ^ { - ( d - 2 + \eta ) } }
\end{array} \Longrightarrow \left\{\begin{array}{l}
\text { class S } \\
\text { class L }
\end{array} \Longrightarrow a=\left\{\begin{array}{c}
1 / z \\
(d-2+\eta) / z
\end{array}\right.\right.\right.
$$

Examples for short-ranged models (class S) include the Ising or Potts models in $d>1$ dimensions (and $T<T_{c}$ ), while all systems quenched to criticality, or the spherical model or the two-dimensional XY model below the Kosterlitz-Thouless transition are examples for long-ranged systems (class L).

The values of these exponents are again collected in table 1 . They have been extensively confirmed in many numerical studies, see [56, 60, 30, 1, 2, 89], and are reproduced in all known analytically solvable models.

In section 2, we recall some of the main facts related to an extension of dynamical scaling towards a local scale-invariance (LSI), first for a dynamical exponent $z=$ 2 and later on possible extensions for $z \neq 2$ and review its application to nondisordered systems. In section 3 we discuss the scaling of responses and correlators in disordered, but non-glassy systems and shall present the available evidence that LSI should be extendable to this class of systems. In section 4 we consider the critical Ising spin glass, present evidence that its non-equilibrium properties depend on the distribution of the coupling constants and show to what extent the data can be explained in terms of LSI. We summarize in section 5.

\section{Local scale-invariance without disorder}

In equilibrium critical phenomena, it is well-known that the standard scale-invariance can, under quite weak conditions, be extended to a conformal invariance. Roughly, a conformal transformation is a scale-transformation $\boldsymbol{r} \mapsto b \boldsymbol{r}$ with a space-dependent rescaling factor $b=b(\boldsymbol{r})$ (such that angles are kept unchanged). In particular, in two dimensions conformal invariance allows to derive from the representation theory of the conformal (Virasoro) algebra the possible values of the critical exponents, to set up a list of possible universality classes, to calculate explicitly all $n$-point correlation functions and so on, see $[14,28,45,53]$. One might wonder whether a similar extension might be possible at least in some instances of dynamical scaling and further ask whether response functions or correlation functions might be found from their covariance under some generalized dynamical scaling with a space-time-dependent rescaling factor $b=b(t, \boldsymbol{r})[52,55]$ ?

We shall first consider the case of phase-ordering where $L(t) \sim t^{1 / z}$ with $z=2$ is known [20] and later describe how this might be generalized to more general values of $z \neq 2$. In particular we enquire what can be said about the scaling functions $f_{C, R}(y)$ in a model-independent way.

A useful starting point is to consider the symmetries of the free diffusion (or free Schrödinger) equation

$$
2 \mathcal{M} \partial_{t} \phi=\Delta \phi
$$

where $\Delta=\nabla \cdot \nabla$ is the spatial laplacian and the 'mass' $\mathcal{M}$ plays the rôle of a kinetic coefficient. Indeed, it was already shown by Lie more than a century ago 
that this equation has more symmetries than the obvious translation- and rotationinvariances. Consider the so-called Schrödinger-group defined through the space-time transformations

$$
t \mapsto t^{\prime}=\frac{\alpha t+\beta}{\gamma t+\delta} ; \boldsymbol{r} \mapsto \boldsymbol{r}^{\prime}=\frac{R \boldsymbol{r}+\boldsymbol{v} t+\boldsymbol{a}}{\gamma t+\delta}, \alpha \delta-\beta \gamma=1
$$

where $\alpha, \beta, \gamma, \delta, \boldsymbol{v}, \boldsymbol{a}$ are real (vector) parameters and $R$ is a rotation matrix in $d$ spatial dimensions. The group acts projectively on a solution $\phi$ of the diffusion equation through $(t, \boldsymbol{r}) \mapsto g(t, \boldsymbol{r}), \phi \mapsto T_{g} \phi$

$$
\left(T_{g} \phi\right)(t, \boldsymbol{r})=f_{g}\left(g^{-1}(t, \boldsymbol{r})\right) \phi\left(g^{-1}(t, \boldsymbol{r})\right)
$$

where $g$ is an element of the Schrödinger group and the companion function reads $[95,100]$

$$
f_{g}(t, \boldsymbol{r})=(\gamma t+\delta)^{-d / 2} \exp \left[-\frac{\mathcal{M}}{2} \frac{\gamma \boldsymbol{r}^{2}+2 R \boldsymbol{r} \cdot(\gamma \boldsymbol{a}-\delta \boldsymbol{v})+\gamma \boldsymbol{a}^{2}-t \delta \boldsymbol{v}^{2}+2 \gamma \boldsymbol{a} \cdot \boldsymbol{v}}{\gamma t+\delta}\right] .
$$

It is then natural to include also arbitrary phase-shifts of the wave function $\phi$ within the Schrödinger group $S c h(d)$. In what follows, we denote by $\mathfrak{s c h}_{d}$ the Lie algebra of $\operatorname{Sch}(d)$. The Schrödinger group so defined is the largest group which maps any solution of the free Schrödinger equation (with $\mathcal{M}$ fixed) onto another solution. For a simple illustration, consider the case $d=1$ and define the Schrödinger operator

$$
\mathcal{S}:=2 M_{0} X_{-1}-Y_{-1 / 2}^{2}
$$

The Schrödinger Lie algebra $\mathfrak{s c h}_{1}=\left\langle X_{-1,0,1}, Y_{-\frac{1}{2}, \frac{1}{2}}, M_{0}\right\rangle$ is spanned by the infinitesimal generators of temporal and spatial translations $\left(X_{-1}, Y_{-1 / 2}\right)$, Galileitransformations $\left(Y_{1 / 2}\right)$, phase shifts $\left(M_{0}\right)$, space-time dilatations with $z=2\left(X_{0}\right)$ and so-called special transformations $\left(X_{1}\right)$. Explicitly, the generators read [52]

$$
\begin{aligned}
& X_{n}=-t^{n+1} \partial_{t}-\frac{n+1}{2} t^{n} r \partial_{r}-\frac{n(n+1)}{4} \mathcal{M} t^{n-1} r^{2}-\frac{x}{2}(n+1) t^{n} \\
& Y_{m}=-t^{m+1 / 2} \partial_{r}-\left(m+\frac{1}{2}\right) \mathcal{M} t^{m-1 / 2} r \\
& M_{n}=-\mathcal{M} t^{n}
\end{aligned}
$$

Here $x$ is the scaling dimension and $\mathcal{M}$ is the mass of the scaling operator $\phi$ on which these generators act. The non-vanishing commutation relations are

$$
\begin{aligned}
& {\left[X_{n}, X_{n^{\prime}}\right]=\left(n-n^{\prime}\right) X_{n+n^{\prime}}, \quad\left[X_{n}, Y_{m}\right]=\left(\frac{n}{2}-m\right) Y_{n+m}} \\
& {\left[X_{n}, M_{n^{\prime}}\right]=-n^{\prime} M_{n+n^{\prime}}, \quad\left[Y_{m}, Y_{m^{\prime}}\right]=\left(m-m^{\prime}\right) M_{m+m^{\prime}}}
\end{aligned}
$$

The invariance of the diffusion equation under the action of $\mathfrak{s c h}_{1}$ is now seen from the following commutators which follow from the explicit form (17)

$$
\begin{aligned}
{\left[\mathcal{S}, X_{-1}\right] } & =\left[\mathcal{S}, Y_{ \pm 1 / 2}\right]=\left[\mathcal{S}, M_{0}\right]=0 \\
{\left[\mathcal{S}, X_{0}\right] } & =-\mathcal{S}, \quad\left[\mathcal{S}, X_{1}\right]=-2 t \mathcal{S}-(2 x-1) M_{0}
\end{aligned}
$$

Therefore, for any solution $\phi$ of the Schrödinger equation $\mathcal{S} \phi=0$ with scaling dimension $x=1 / 2$, the infinitesimally transformed solution $\mathcal{X} \phi$ with $\mathcal{X} \in \mathfrak{s c h}_{1}$ also 
satisfies the Schrödinger equation $\mathcal{S} \mathcal{X} \phi=0$ [80, 95, 51]. For applications to ageing, we must consider to so-called ageing algebra $\mathfrak{a g e}_{1}=\left\langle X_{0,1}, Y_{-\frac{1}{2}, \frac{1}{2}}, M_{0}\right\rangle \subset \mathfrak{s c h}_{1}$ (without time-translations) which is a true subalgebra of $\mathfrak{s c h}_{1}$. Extensions to $d>1$ are straightforward.

What is the usefulness of knowing dynamical symmetries of free, simple diffusion for the understanding of non-equilibrium kinetics ? One way of setting up the problem would be to write down a stochastic Langevin equation for the order-parameter. The simplest case is usually considered to be a dynamics without macroscopic conservation laws (model A), where one would have [71]

$$
2 \mathcal{M} \frac{\partial \phi}{\partial t}=\Delta \phi-\frac{\delta \mathcal{V}[\phi]}{\delta \phi}+\eta
$$

where $\mathcal{V}$ is the Ginzburg-Landau potential and $\eta$ is a gaussian noise which describes the coupling to an external heat-bath and the initial distribution of $\phi$. At first sight, there appear to be no non-trivial symmetries, since the noise term $\eta$ is incompatible with a Galilei-invariant equation (20). To understand this physically, consider a magnet which is at rest with respect to a homogeneous heat-bath at temperature $T$. If the magnet is moved with a constant velocity with respect to the heat-bath, the effective temperature will now appear to be direction-dependent, and the heat-bath is no longer homogeneous. However, that difficulty can be avoided as follows [103]: split the Langevin equation into a 'deterministic' part with non-trivial symmetries and a 'noise' part and then show using these symmetries that all averages can be reduced exactly to averages within the deterministic, noiseless theory. Technically, one first constructs in the standard fashion (Janssen-de Dominicis procedure) $[36,77]$ the associated stochastic field-theory with action $J[\phi, \widetilde{\phi}]$ where $\widetilde{\phi}$ is the response field associated to the order-parameter $\phi$. Second, decompose the action into two parts

$$
J[\phi, \widetilde{\phi}]=J_{0}[\phi, \widetilde{\phi}]+J_{b}[\widetilde{\phi}]
$$

where

$$
J_{0}[\phi, \widetilde{\phi}]=\int_{\mathbb{R}_{+} \times \mathbb{R}^{d}} \mathrm{~d} t \mathrm{~d} \boldsymbol{r} \widetilde{\phi}\left(2 \mathcal{M} \partial_{t} \phi-\Delta \phi+\frac{\delta \mathcal{V}}{\delta \phi}\right)
$$

contains the terms coming from the 'deterministic' part of the Langevin equation $(\mathcal{V}$ is the self-interacting 'potential') whereas

$$
J_{b}[\widetilde{\phi}]=-T \int_{\mathbb{R}_{+} \times \mathbb{R}^{d}} \mathrm{~d} t \mathrm{~d} \boldsymbol{r} \widetilde{\phi}(t, \boldsymbol{r})^{2}-\frac{1}{2} \int_{\mathbb{R}^{2 d}} \mathrm{~d} \boldsymbol{r} \mathrm{d} \boldsymbol{r}^{\prime} \widetilde{\phi}(0, \boldsymbol{r}) a\left(\boldsymbol{r}-\boldsymbol{r}^{\prime}\right) \widetilde{\phi}\left(0, \boldsymbol{r}^{\prime}\right)
$$

contains the terms coming either from the noisy coupling to the heat bath or else from the average over the disordered initial conditions [77]. It was assumed here that $\langle\phi(0, \boldsymbol{r})\rangle=0$ and $a(\boldsymbol{r})$ denotes the initial two-point correlator

$$
a(\boldsymbol{r}):=C\left(0,0 ; \boldsymbol{r}+\boldsymbol{r}^{\prime}, \boldsymbol{r}^{\prime}\right)=\left\langle\phi\left(0, \boldsymbol{r}+\boldsymbol{r}^{\prime}\right) \phi\left(0, \boldsymbol{r}^{\prime}\right)\right\rangle=a(-\boldsymbol{r})
$$

while the last relation follows from spatial translation-invariance which we shall admit throughout. Averages are of course calculated from the functional integral

$$
\langle\mathcal{A}\rangle=\int \mathcal{D} \phi \mathcal{D} \widetilde{\phi} \mathcal{A}[\phi, \widetilde{\phi}] e^{-J[\phi, \widetilde{\phi}]} .
$$

It is instructive to consider briefly the case of a free field, where $\mathcal{V}=0$. Variation of (21) with respect to $\widetilde{\phi}$ and $\phi$, respectively, then leads to the equations of motion 


$$
2 \mathcal{M} \partial_{t} \phi=\Delta \phi+T \widetilde{\phi},-2 \mathcal{M} \partial_{t} \widetilde{\phi}=\Delta \widetilde{\phi}
$$

The first one of those might be viewed as a Langevin equation if $\widetilde{\phi}$ is interpreted as a noise. Comparison of the two equations of motion (26) shows that if the orderparameter $\phi$ is characterized by the 'mass' $\mathcal{M}$ (which by physical convention is positive), then the associated response field $\widetilde{\phi}$ is characterized by the negative mass $-\mathcal{M}$. This characterization remains valid beyond free fields.

We now concentrate on actions $J_{0}[\phi, \widetilde{\phi}]$ which are Galilei-invariant. This means that if $\langle.\rangle_{0}$ denotes the averages calculated only with the action $J_{0}$, the Bargman superselection rules [7]

$$
\langle\underbrace{\phi \ldots \phi}_{n} \underbrace{\widetilde{\phi} \ldots \widetilde{\phi}}_{m}\rangle_{0} \sim \delta_{n, m}
$$

hold true. It follows that both response and correlation functions can be exactly expressed in terms of averages with respect to the deterministic part alone. For example (we suppress for notational simplicity the spatial coordinates) [103]

$$
R(t, s)=\left.\frac{\delta\langle\phi(t)\rangle}{\delta h(s)}\right|_{h=0}=\langle\phi(t) \widetilde{\phi}(s)\rangle=\left\langle\phi(t) \widetilde{\phi}(s) e^{-J_{b}[\widetilde{\phi}]}\right\rangle_{0}=\langle\phi(t) \widetilde{\phi}(s)\rangle_{0}
$$

where the 'noise' part of the action was included in the observable and the Bargman superselection rule (27) was used. In other words, the two-time response function $R(t, s)=R_{0}(t, s)$ does not depend explicitly on the 'noise' at all! The correlation function is reduced similarly [103]

$$
\begin{aligned}
& C(t, s ; \boldsymbol{r})=T \int_{\mathbb{R}_{+} \times \mathbb{R}^{d}} \mathrm{~d} u \mathrm{~d} \boldsymbol{R}\left\langle\phi(t, \boldsymbol{r}+\boldsymbol{y}) \phi(s, \boldsymbol{y}) \widetilde{\phi}(u, \boldsymbol{R})^{2}\right\rangle_{0} \\
& +\frac{1}{2} \int_{\mathbb{R}^{2 d}} \mathrm{~d} \boldsymbol{R} \mathrm{d} \boldsymbol{R}^{\prime} a\left(\boldsymbol{R}-\boldsymbol{R}^{\prime}\right)\left\langle\phi(t, \boldsymbol{r}+\boldsymbol{y}) \phi(s, \boldsymbol{y}) \widetilde{\phi}(0, \boldsymbol{R}) \widetilde{\phi}\left(0, \boldsymbol{R}^{\prime}\right)\right\rangle_{0} .
\end{aligned}
$$

Only terms which depend explicitly on the 'noise' remain - recall the vanishing of the 'noiseless' two-point function $\langle\phi(t) \phi(s)\rangle_{0}=0$ because of the Bargman superselection rule. Using mathematical methods explained in [57] and restricting to a disordered initial state $a(\boldsymbol{r})=a_{0} \delta(\boldsymbol{r})$, the resulting three-point function can be calculated. It is satisfying that $\lambda_{C}=\lambda_{R}$ follows [103], in agreement with an earlier derivation [20].

Therefore, the dynamical symmetries of non-equilibrium kinetics are characterized by the 'deterministic' part of Langevin equation. This result only depends on the Galilei-invariance of the 'deterministic' part (provided that $\phi$ and $\widetilde{\phi}$ transform projectively through eq. (14)). Clearly, linear equations are Galilei-invariant in this sense. On the other hand, the question is considerably more complicated for nonlinear equations. For the purposes of this review it is sufficient to state that deterministic non-linear diffusion/Schrödinger equations with $\mathfrak{a g} \mathfrak{e}_{1}$ or $\mathfrak{s c h}_{1}$ as a dynamical symmetry and sufficiently general to be applicable to phase-ordering kinetics have been explicitly constructed $[115,9]$. In particular, it has been shown that independently of the form of the potential the same representation of $\mathfrak{a g e}_{d}$ or $\mathfrak{s c h}{ }_{d}$ applies, in agreement with universality. We refer to the literature for the details.

Since all quantities of interest will reduce to some kind of response function, one may calculate them from the requirement that they transform covariantly under the action ageing subgroup (with Lie algebra $\mathfrak{a g} \mathfrak{e}_{d}$ ) obtained from the Schrödinger group when leaving out time-translations. We shall concentrate here on the two-time 
autoresponse function $R(t, s)=\langle\phi(t) \widetilde{\phi}(s)\rangle$ built from so-called quasiprimary [14] scaling operators $\phi$ and $\widetilde{\phi}$ which transform according to the generators of the ageing algebra. Then the requirement of covariance of $R$ reduces to the two conditions $X_{0} R(t, s)=X_{1} R(t, s)=0$. Since time-translations are not included in the ageing group, the generators $X_{n}$ can be generalized from (17) to the following form

$$
X_{n}=-t^{n+1} \partial_{t}-\frac{n+1}{2} t^{n} r \partial_{r}-\frac{(n+1) n}{4} \mathcal{M} t^{n-1} r^{2}-\frac{x}{2}(n+1) t^{n}-\xi n t^{n} ; n \geq 0
$$

where $\xi$ is a new quantum number associated with the field $\phi$ on which the generators $X_{n}$ act. Consequently, a quasiprimary scaling operator which transforms covariantly under the ageing algebra is characterized by the triplet $(x, \xi, \mathcal{M})$. The last term in (30) can only be present for systems out of an equilibrium state. In particular, the requirement of time-translation invariance and $\left[X_{1}, X_{-1}\right]=2 X_{0}$ lead to $\xi=0$ and any representation of $\mathfrak{s c h}_{d}$ must have $\xi=0$. In addition, the last commutator in (19) must be replaced by $\left[\mathcal{S}, X_{1}\right]=-2 t \mathcal{S}-(2 x+2 \xi-1) M_{0}$. The meaning of $\xi$ can be understood by integrating the generators $X_{n}$ in order to recover the finite, noninfinitesimal transformations. Then it follows that if $\xi \neq 0$, lattice observables are related to the quasiprimary fields $\Phi$ of ageing invariance according to $\phi_{\text {lattice }}(t) \mapsto$ $\mathfrak{a}^{-x} t^{\xi} \Phi(t)$ with an additional time-dependent factor [65] and where $\mathfrak{a}$ is a lattice constant. Rather than being an exotic exception, this extension of the Schrödinger transformations seems to occur quite generically, the best-known examples being the one-dimensional Glauber-Ising model at zero temperature. For further examples, see table 3 below. Finally, solving the two differential equations for $R$ gives the explicit form of $R(t, s)$, see (34) below.

While this discussion was carried out explicitly for the case $z=2$, it is tempting to try and generalize this idea to more general values of $z$. In this way, the notion of local scale-transformation has been introduced, which is based on the following main assumptions [55].

1. In principle, the following conformal time-transformations should be included

$$
t \mapsto t^{\prime}=\frac{\alpha t+\beta}{\gamma t+\delta} ; \alpha \delta-\beta \gamma=1
$$

For applications to ageing, however, time-translations generated by $\beta$ must be left out (generalizing the restriction $\mathfrak{s c h}_{d} \rightarrow \mathfrak{a g e}_{d}$ ).

2. The generator $X_{0}$ of scale-transformations is

$$
X_{0}=-t \partial_{t}-\frac{1}{z} r \partial_{r}-\frac{x}{z}
$$

where $x$ is the scaling dimension of the quasi-primary operator on which $X_{0}$ is supposed to act. Physically, this implies that there is a single relevant length scale $L(t) \sim t^{1 / z}$

3. Spatial translation-invariance is required.

Generators for infinitesimal local scale-transformations have been explicitly constructed [55]. In almost all cases, however, it turned out that terms containing certain fractional derivatives are needed. Skipping over the details of their construction which can be found in the literature, it is sufficient here to state that one can show that the linear equation $\mathcal{S}_{z} \phi=0$ with the generalized Schrödinger operator 


$$
\mathcal{S}_{z}=-\lambda \partial_{t}+\left(\nabla_{\boldsymbol{r}}^{2}\right)^{z / 2}
$$

where $\lambda$ is a constant, satisfies with the generators of local scale-transformations commutation relations quite similar to eq. (19) which establishes local scale-invariance as a dynamical symmetry of that linear equation [55, 110, 12]. Attempts to suppress the fractional terms in the generators of local scale-transformations lead either to $\lambda \rightarrow 0$ or to $\lambda \rightarrow \infty$ (there is an exception if $z=1$ [55]). Explicit tests have recently been performed for $z=4$ in models of kinetic growth described by the Mullins-Herring equation [110] and the kinetic spherical model with conserved order-parameter [12]. Further tests in long-ranged models where $z$ can be continuously tuned through a control parameter are currently being carried out [13]. On the other hand, a systematic derivation of an analogue of the Bargman superselection rules for $z \neq 2$ is still lacking and indeed constitutes one of the most important open questions to be overcome for a further development of the theory.

Local scale-invariance (LSI) assumes in particular that the two-time response functions transform covariantly under these local scale-transformations, hence $X_{0} R=$ $X_{1} R=0$. This leads to the following prediction for the autoresponse [55, 103, 62, 65]

$$
R(t, s)=\langle\phi(t) \widetilde{\phi}(s)\rangle=s^{-1-a} f_{R}(t, s), f_{R}(y)=f_{0} y^{1+a^{\prime}-\lambda_{R} / z}(y-1)^{-1-a^{\prime}}
$$

where the exponents $a, a^{\prime}, \lambda_{R} / z$ are related to $x, \xi, \widetilde{x}, \widetilde{\xi}$ and $f_{0}$ is a normalization constant. ${ }^{5}$ Spatio-temporal responses can be found similarly, with the result

$$
R(t, s ; \boldsymbol{r})=\left.\frac{\delta\langle\phi(t, \boldsymbol{r})\rangle}{\delta h(s, \mathbf{0})}\right|_{h=0}=R(t, s) \Phi\left(|\boldsymbol{r}|(t-s)^{-1 / z}\right)
$$

where $\Phi(u)=\exp \left(-\frac{1}{2} \mathcal{M} u^{2}\right)$ if $z=2$. For $z \neq 2$, the function $\Phi(u)$ has to be found from a known fractional differential equation [55, 110, 12].

Starting with [54], the prediction (34) has been reproduced in many different spin systems and we list examples quenched to below criticality in table 2 and quenched to the critical point in table 3 . For $T<T_{c}$, it is found empirically that $a=a^{\prime}$ in all examples considered so far. ${ }^{6}$ We point out that agreement with local scale-invariance eq. (34) is not only obtained for systems where the dynamical exponent is $z=2$, but that rather there exist quite a few examples where $z$ can become considerably larger or smaller than 2. It must be remembered, however, that the above derivation of (34) for a stochastic Langevin equation has for the time being only been carried out for $z=2$ and the justification of $X_{0} R=X_{1} R=0$ remains an open problem for $z \neq 2$ in general although the result eq. (34) seems to work remarkably well. It is non-trivial that a relatively simple extension of dynamical scaling should be capable of making predictions which can be reproduced in physically quite different systems.

A few comments are still needed: (i) for the XY model in the spin-wave approximation (table 2), eq. (34) holds for the response of the angular variable $\phi=\phi(t, \boldsymbol{r})$ which is related to the XY spin through $\boldsymbol{S}=(\cos \phi, \sin \phi)$. Magnetic responses have

\footnotetext{
${ }^{5}$ We point out that the prediction (34) as well as the explicit form (30) of $X_{n}$, valid for $z=2$, assume that the mean order-parameter $\langle\phi(0, \boldsymbol{r})\rangle=m_{0}=0$ at the initial moment when the quench to $T<T_{c}$ or $T=T_{c}$ is made.

${ }^{6}$ Recall that $M_{\mathrm{TRM}}(t, s)$ in direct space is not very sensitive to $a-a^{\prime}$ and that most numerical studies were carried out in this setting. A considerably more sensitive test looks at $M_{\mathrm{TRM}}$ in momentum space [104].
} 
Table 2. Magnetic systems quenched into the coexistence phase $\left(T<T_{c}\right)$ which satisfy (34) with the exponents $a=a^{\prime}$ and $\lambda_{R} . d$ is the spatial dimension and the numbers in brackets estimate the numerical uncertainty in the last digit(s). In the spherical model, long-range initial conditions are included and in the longrange spherical model the exchange couplings decay as $J_{\boldsymbol{r}} \sim|\boldsymbol{r}|^{-d-\sigma}$. In the bonddisordered Ising model, the couplings are taken homogeneously from the interval $[1-\varepsilon / 2,1+\varepsilon / 2]$. Then $z=z(T, \varepsilon)=2+\varepsilon / T$ [97] and one observes roughly $1.3 \lesssim$ $\lambda_{R}(T, \varepsilon) \lesssim 1.7$.

\begin{tabular}{||l|rr|rr|l|l||}
\hline \hline \hline model & $d$ & $z$ & $a=a^{\prime}$ & $\lambda_{R}$ & & Ref. \\
\hline \hline Ising & 2 & 2 & $1 / 2$ & $1.26(1)$ & & {$[58]$} \\
& 2 & 2 & $\simeq 0.5$ & $1.24(2)$ & & {$[89,75]$} \\
& 3 & 2 & $1 / 2$ & $1.60(2)$ & & {$[58]$} \\
\hline Potts-3 & 2 & 2 & 0.49 & $1.19(3)$ & & {$[89,75]$} \\
\hline Potts-8 & 2 & 2 & 0.51 & $1.25(1)$ & & {$[89,75]$} \\
\hline XY & 3 & 2 & 0.5 & 1.7 & & {$[2]$} \\
XY spin wave & $\geq 2$ & 2 & $d / 2-1$ & $d$ & angular response & {$[103]$} \\
\hline spherical & $>2$ & 2 & $d / 2-1(d-\alpha) / 2$ & $C_{\text {ini }}(\boldsymbol{r}) \sim|\boldsymbol{r}|^{-d-\alpha}$ & {$[94,102]$} \\
\hline long-range & $>2$ & $\sigma$ & $d / \sigma-1$ & $d / 2$ & $0<\sigma<2$ & \\
spherical & $\leq 2$ & $\sigma$ & $d / \sigma-1$ & $d / 2$ & $0<\sigma<d$ & {$[27]$} \\
\hline \hline random-bond Ising & $22+\varepsilon / T$ & $1 / z(T, \varepsilon)$ & $\lambda_{R}(T, \varepsilon)$ & disordered & {$[66]$} \\
\hline \hline
\end{tabular}

a different scaling form $[17,1]$. (ii) In the critical disordered Ising model (table 3) one finds a logarithmic scaling form $R(t, s)=\left(r_{0}+r_{1} \ln (t-s)\right) f_{R}(t / s)[24,111,112]$ such that the computed $f_{R}(y)$ is consistent with (34) to one-loop order, or up to terms of order $\mathrm{O}(\varepsilon)$. (iii) Finally, a two-loop calculation of the critical non-conserved $\mathrm{O}(n)$-model does produce in $4-\varepsilon$ dimensions an expression for $f_{R}(y)$ which is incompatible with (34) [23] and a similar result is anticipated in $2+\varepsilon$ dimensions [43], although the one-loop results are still compatible [22, 23, 43]. Should one conclude from these studies that for $T=T_{c}$ the prediction (34) and by implication local scale-invariance can only hold approximatively ? This might well be a subtle question. Deviations between (34) and the field-theoretical studies typically arise when $t / s \approx 1$. However, in this region the field-theoretical results for $f_{R}(y)$ do not agree with the ones of non-perturbative numerical studies [104]. Since the perturbative expansion usually carried out in field-theoretical studies does not necessarily take care of the Galilei-invariance, it is necessary to carefully check that the truncation of the $\varepsilon$-series does not introduce slight inaccuracies. Only after this has been done (for example by re-summing the $\varepsilon$-series) and checked by comparing with non-perturbative data, meaningful quantitative statements on the scaling functions can be made. (iv) Throughout, it was implicitly assumed that the order-parameter vanishes initially. Systematic studies on what happens when this condition is relaxed are only now becoming available $[6,26,43,11]$. These extensions might be particularly important for chemical kinetics [67] and the existing simulations for directed percolation (or the contact process) may well turn out to be an example where the initial non-vanishing 
Table 3. Systems quenched to a critical point of their stationary state which satisfy (34) with the exponents $a, a^{\prime}$ and $\lambda_{R} / z . d$ is the spatial dimension and the numbers in brackets estimate the uncertainty in the last digit(s). CSM stands for the spherical model with a conserved order-parameter, FA denotes the Frederikson-Andersen model, NEKIM is the non-equilibrium kinetic Ising model and BCP and BPCP denote the bosonic contact and pair-contact processes, respectively. In the BPCP dynamical scaling only holds along a part of the critical line. In the spherical model, long-range initial correlations $C_{\text {ini }}(\boldsymbol{r}) \sim|\boldsymbol{r}|^{-d-\alpha}$ were considered. If $d+\alpha>2$, these reduce to short-ranged initial correlations (denoted $\mathrm{s}$ ), but for $d+\alpha<2$ a new class $\mathrm{L}$ arises. In those models described by a Langevin equation, the simple white noise $\left\langle\eta(t, \boldsymbol{r}) \eta\left(s, \boldsymbol{r}^{\prime}\right)\right\rangle=2 T \delta\left(\boldsymbol{r}-\boldsymbol{r}^{\prime}\right) \delta(t-s)$ was used, with the only exception of the CSM. In the Ising spin glass, a bimodal disorder was used.

\begin{tabular}{|c|c|c|c|c|c|c|}
\hline model & $d$ & $\bar{a}$ & $\overline{a^{\prime}-a}$ & $\lambda_{R} / z$ & & $\overline{\text { Ref. }}$ \\
\hline random walk & & -1 & 0 & 0 & & [35] \\
\hline OJK-model & & $(d-1) / 2$ & $-1 / 2$ & $d / 4$ & & {$[16,92,62]$} \\
\hline Ising & $\begin{array}{l}1 \\
2 \\
3\end{array}$ & $\begin{array}{r}0 \\
0.115 \\
0.506\end{array}$ & $\begin{array}{r}-1 / 2 \\
-0.187(20) \\
-0.022(5)\end{array}$ & $\begin{array}{r}1 / 2 \\
0.732(5) \\
1.36\end{array}$ & & $\begin{array}{l}{[47,88,59]} \\
{[104,65]} \\
{[104,65]}\end{array}$ \\
\hline $\mathrm{XY}$ & 3 & 0.52 & 0 & $1.34(5)$ & & {$[2]$} \\
\hline spherical $d>2$ & $\begin{array}{l}<4 \\
>4 \\
<4 \\
>4\end{array}$ & $\begin{array}{l}d / 2-1 \\
d / 2-1 \\
d / 2-1 \\
d / 2-1\end{array}$ & $\begin{array}{l}0 \\
0 \\
0 \\
0\end{array}$ & $\begin{array}{r}\alpha / 4-1 / 2 \\
\alpha) / 4+1 / 2 \\
3 d / 4-1 \\
d / 2\end{array}$ & $\begin{array}{l}\mathrm{L} \\
\mathrm{L} \\
\mathrm{S} \\
\mathrm{S} \\
\end{array}$ & $\begin{array}{l}{[102]} \\
{[102]} \\
{[48]} \\
{[48]}\end{array}$ \\
\hline $\mathrm{CSM}$ & $>2$ & $d / 4-1$ & 0 & $(d+2) / 4$ & & {$[12]$} \\
\hline disordered Ising & $4-\varepsilon$ & $1-\frac{1}{2} \sqrt{\frac{6 \varepsilon}{53}}$ & 0 & $3-\frac{1}{2} \sqrt{\frac{6 \varepsilon}{53}}$ & $\mathrm{O}(\varepsilon), \log$ & {$[24,111,112]$} \\
\hline FA & $\begin{array}{r}>2 \\
1\end{array}$ & $\begin{array}{r}1+d / 2 \\
1\end{array}$ & $\begin{array}{r}-2 \\
-3 / 2 \\
\end{array}$ & $\begin{array}{r}2+d / 2 \\
2 \\
\end{array}$ & & $\begin{array}{l}{[91]} \\
{[91,90]}\end{array}$ \\
\hline Ising spin glass & 3 & $0.060(4)$ & $-0.76(3)$ & $0.38(2)$ & see sect. 4 & {$[62,63]$} \\
\hline contact process & $\begin{array}{r}1 \\
>4 \\
\end{array}$ & $\begin{array}{l}-0.681 \\
d / 2-1\end{array}$ & $\begin{array}{r}+0.270(10) \\
0\end{array}$ & $\begin{array}{l}1.76(5) \\
d / 2+2\end{array}$ & $t / s \gtrsim 1.1$ & $\begin{array}{l}{[41,70,65]} \\
{[106]}\end{array}$ \\
\hline NEKIM & 1 & $-0.430(4)$ & $\overline{0}$ & $1.9(1)$ & & [96] \\
\hline $\mathrm{BCP}$ & $\geq 1$ & $d / 2-1$ & $\overline{0}$ & $d / 2$ & & {$[8,9]$} \\
\hline $\mathrm{BPCP}$ & $>2$ & $d / 2-1$ & 0 & $d / 2$ & $\alpha \leq \alpha_{C}$ & {$[8,9]$} \\
\hline
\end{tabular}


value of the order-parameter influences the form of scaling functions such as $f_{R}(y)$ $[11,41,106,70]$. From that point of view it is surprising that eq. (34) could describe any part of the data of $f_{R}(y)$ as well as it does. (v) We did not include growth models since their analysis from the point of view of LSI is just beginning $[110,12]$.

If $z=2$, it is also possible, using eq. (29), to derive explicit predictions for the two-time correlation function [103, 61]. These have been tested in some exactly solvable models [103, 65], the two-dimensional Ising model [61] and the two-dimensional $q$-states Potts model with $q=2,3,8[89,75]$. Extensions to $z=4$ have been studied very recently $[110,12]$.

Finally, let us mention that the prediction (35) for the space-time response has been verified for $z=2$ through numerical simulations of two- and three-dimensional Ising models undergoing phase-ordering [60] and from the exact solutions of the $1 D$ Glauber-Ising model $[47,88,59]$ at $T=0$, the spherical model in $d>2$ dimensions $[48,102]$ and the long-range spherical model $[27,13]$.

\section{Disordered ferromagnets}

We now take up the discussion of disordered systems. Rather than going directly to spin glasses, it may be useful to consider first disordered, but not frustrated systems, such that the random exchange couplings $J_{i, j} \geq 0$ in models such as (4).

We point out that a disorder in the exchange couplings, as introduced in the random-bond model (4), can have quite different consequences than a random site dilution, as described by a classical hamiltonian $\mathcal{H}=-J \sum_{(i, j)} \varepsilon_{i} \varepsilon_{j} \sigma_{i} \sigma_{j}$ where $\varepsilon_{i} \in$ $\{0,1\}$ are random variables selected according to a control parameter $p$. Provided that on average $\overline{J_{i, j}}=1$, the critical temperature in the presence of bond disorder will not change much. For a site-diluted model, however, the phase-transition should disappear once the non-magnetic sites (where $\varepsilon_{i}=0$ ) will begin to percolate. Here, we shall mainly restrict attention to random-bond disorder and shall furthermore restrict to the case of quenched, that is immobile, disorder.

The first question to be addressed concerns the existence and the detailed form of dynamical scaling, as expressed through the time-dependence $L=L(t)$ of the linear size of the clusters. The physical picture is as follows [72], see also the middle column of figure 3. Initially, small clusters will form and start to grow. These early stages are still unaffected by the presence of the disorder which will only begin to be felt once the average cluster size has become of the same order as the mean distance between two impurities. Then the domain walls will become trapped close to the disorder-induced defects and their motion will slow down correspondingly. Rather than being described by purely geometric considerations as it can be done through the Allen-Cahn equation for non-disordered systems, the impurities act as energy barriers to the domain growth, hence the pinning centers are localized in energetically favourable positions, which explains the slowing-down of the kinetics. Phenomenologically, for a non-conserved order-parameter one still expects that the basic curvature-reducing mechanism is applicable, leading to [72, 84]. This yields

$$
\frac{\mathrm{d} L(t)}{\mathrm{d} t}=\frac{D(L, T)}{L(t)}
$$

where the diffusion constant $D=D(L, T)$ now depends on the domain scale $L=L(t)$ and the temperature $T$. For a constant $D$, one is back to the non-disordered case, 
with $L(t) \sim t^{1 / 2}$. For thermally activated motion, one should have

$$
D(L, T) \simeq D_{0} \exp \left(-E_{B} / T\right)
$$

where $E_{B}=E_{B}(L)$ is the barrier energy. Huse and Henly [72] have argued long ago that the barrier energy should depend on the domain size algebraically $E_{B}(L) \simeq$ $E_{0} L^{\psi}$, where the exponent $\psi=(2 \zeta+d-3) /(2-\zeta)$ where $\zeta$ is the domain-wall roughness exponent. For example, in two dimensions it is known that $\zeta=\frac{2}{3}$, hence $\psi=\frac{1}{4}$. Inserting into eq. (36) leads to [72]

$$
L(t)=\left(\frac{T}{E_{0}}\right)^{1 / \psi} \mathcal{L}\left(\frac{t}{t_{0}}\right), \quad \mathcal{L}(\tau)= \begin{cases}\frac{2}{\psi} \tau & ; \tau \ll 1 \\ (\ln \tau)^{1 / \psi} & ; \tau \gg 1\end{cases}
$$

which describes the qualitative change of behaviour between the initial regime and a second regime of slower growth for larger times.

Table 4. Experimental results on the growth of the domain size $L(t)$ in some disordered, but non-glassy systems in two dimensions. For $\mathrm{Rb}_{2} \mathrm{Co}_{0.60} \mathrm{Mg}_{0.40} \mathrm{~F}_{4}$ a different temperature-dependent exponent $\psi$ is found close to $T_{c}$. For TGS the order-parameter

\begin{tabular}{|c|c|c|c|c|c|}
\hline material & model & $L(t)$ & $\psi$ & $\left.T_{c}[K]\right]$ & Ref. \\
\hline $\mathrm{Rb}_{2} \mathrm{Co}_{0.60} \mathrm{Mg}_{0.40} \mathrm{~F}_{4}$ & $\begin{array}{l}\text { diluted Ising } \\
\text { antiferromagnet }\end{array}$ & $\overline{A+B(\ln t)^{1 / \psi}}$ & $\begin{array}{r}0.28(15[K] / T) \\
\text { if } T \lesssim 15[K]\end{array}$ & 20 & {$[74]$} \\
\hline $\begin{array}{l}\text { tryglycine sulfate } \\
\text { (TGS) }\end{array}$ & $\begin{array}{l}\text { ferroelectric } \\
\text { conserved }\end{array}$ & $\begin{array}{l}\sim\left(t-t_{0}\right)^{1 / z} \\
\sim(\ln t)^{1 / \psi}\end{array}$ & $\begin{array}{l}z \approx 3 \\
1 / 4\end{array}$ & 322 & {$[86,87]$} \\
\hline $\mathrm{Rb}_{2} \mathrm{Cu}_{0.89} \mathrm{Co}_{0.11} \mathrm{~F}_{4}$ & $\begin{array}{l}\text { random-bond } \\
\text { Ising }\end{array}$ & $(\ln t)^{1 / \psi}$ & $\begin{array}{l}0.20(5) \\
\quad T=3.9[K]\end{array}$ & $4.93(5)$ & {$[114]$} \\
\hline $\begin{array}{l}\text { ZLI } 4792 \text { (Merck) } \\
\text { (liquid crystal) }\end{array}$ & $\begin{array}{l}\text { random-bond } \\
\text { Ising }\end{array}$ & $(\ln t)^{1 / \psi}$ & $1 / 4$ & & {$[113]$} \\
\hline
\end{tabular}
is conserved.

We now compare this result with the available experimental evidence collected in table 4. Although there is a general qualitative agreement about a a change from a relatively fast kinetics seen at not too late times $[74,86,87]$ to slowing-down of the kinetics at later times, quantitatively the situation remains a little ambiguous. In the published work known to us, data were usually compared to a logarithmic law $L(t) \sim(\ln t)^{1 / \psi}$, which rather than being an objective statement might simply come from the fact that the prediction (38) of Huse and Henley [72] has been around for quite some time. On the other hand, there is some experimental information which is not consistent with eq. (38), if it can be taken at face value. For example, in at least one system the effective exponent depends on temperature [74] while a constant $\psi=\frac{1}{4}$ is theoretically expected in two dimensions [72]. Indeed, it has been argued that in site-diluted Ising models, because of the fractal nature of the domains boundaries the energy barriers should rather depend logarithmically on $L(t)$, viz. $E_{B}(L)=\varepsilon \ln (1+L)[69,107,97]$. Inserting this into (36) leads to an algebraic law $L(t) \sim t^{1 / z}$ where the effective $z$ crosses over from $z=\frac{1}{2}$ for short times to 


$$
z=z(T, \varepsilon)=2+\varepsilon / T
$$

for late times. In at least one experiment, data were explicitly seen to be also compatible with an algebraic growth law [86, 87].

For a conserved order-parameter, a similar discussion can be carried out. It can be shown that for algebraic energy barriers the result (38) remains unchanged [72], whereas for logarithmic barriers one finds again algebraic growth $L(t) \sim t^{1 / z}$ with $z=z(T, \varepsilon)=3+\varepsilon / T[97,98]$.

Theoretical work has among others studied a random-bond Ising model defined by the classical hamiltonian

$$
\mathcal{H}_{\mathrm{dis}}=-\sum_{(i, j)} J_{i, j} \sigma_{i} \sigma_{j}
$$

where the $J_{i, j}$ are random variables uniformly distributed over the interval [ 1 $\varepsilon / 2,1+\varepsilon / 2]$ and where a non-conserved dynamics was created via a Metropolis or heat-bath algorithm. For examples, estimates for $L(t)$ extracted from the singletime spatio-temporal correlation function were seen to be in full agreement with the prediction (39) of logarithmic energy barriers [97, 98]. The same conclusions are obtained from the dynamical scaling behaviour of two-time quantities [66], see also below. It would be desirable to compare existing experimental data with the possibility of logarithmic energy barriers. If the discrepancy between the information extracted from experiments and theoretical simulations should persist, it could mean that the usually considered theoretical models are not realistic enough for a quantitative description of real disordered materials.

We here discuss the thermoremanent magnetization $M_{T R M}(t, s)$ which is obtained when quenching the system in presence of a small magnetic field. The magnetic field is cut after the waiting time $s$ and the decay of the magnetization is then monitored as a function of time. The thermoremanent magnetization is related to the response $R(t, s)$ through

$$
M_{\mathrm{TRM}}(t, s)=h_{0} \int_{0}^{s} \mathrm{~d} u R(t, u),
$$

where $h_{0}$ is the amplitude of the small magnetic field. The interest in this integrated response function comes from the fact that a direct calculation of the functional derivative which defines $R$ produces extremely noisy data, in contrast to the measurement of $M_{T R M}$ where the noise is to a large extend smoothed out by the integration.

From our experience with the scaling of $M_{\mathrm{TRM}}(t, s)$ in the phase-ordering of simple magnets, we expect the scaling behaviour $[56,60]$

$$
M_{\mathrm{TRM}}(t, s)=r_{0} s^{-a} f_{M}(t / s)+r_{1} s^{-\lambda_{R} / z} g_{M}(t / s), g_{M}(y) \simeq y^{-\lambda_{R} / z} .
$$

Note that we have here included the leading correction term $\sim s^{-\lambda_{R} / z} g_{M}(t / s)$ which can become quite sizeable in systems undergoing phase-ordering and which must be subtracted off before a reliable determination of the scaling function $f_{M}(y)$ is possible. In the framework of LSI an analytical expression for $f_{M}(y)$ is readily derived from (34) with $a=a^{\prime}$ and reads $\left({ }_{2} F_{1}\right.$ is a hypergeometric function)

$$
f_{M}(y)=y^{-\lambda_{R} / z} F_{1}\left(1+a, \frac{\lambda_{R}}{z}-a ; \frac{\lambda_{R}}{z}-a+1 ; \frac{1}{y}\right) .
$$


The expected scaling behaviour (42) of the linear response has been studied through large-scale numerical simulations [66]. As an example, figure 4 shows data obtained for $\varepsilon=0.5$ and $T=1$. This is a case where the finite-time correction to the leading behaviour $\sim s^{-a} f_{M}(t / s)$ is negligible, i.e. $r_{1} \approx 0$ in eq. (42). Plotting $M_{\mathrm{TRM}}(y s, s)$ over against $s$ in a log-log plot nicely verifies the expected scaling behaviour and yields in addition an estimate for the ageing exponent $a$, see table 5 . It is worth noting that the estimates are in complete agreement with the relation $a(T, \epsilon)=1 / z(T, \epsilon)$ expected for systems of class $S$ as discussed in section 1 , where the dynamical exponent $z$ is given by (39). This confirms the conclusion of $[97,98]$ that the random-bond Ising model shows simple ageing and furthermore $L(t) \sim t^{1 / z}$ with $z=z(T, \epsilon)$ given by eq. (39).

We also compiled in table 5 the estimates [66] for the non-universal constants $r_{0}$ and $r_{1}$ as well as for the exponent $\lambda_{R} / z$ which governs the asymptotic behaviour of the scaling function $f_{M}(y) \sim y^{-\lambda_{R} / z}$.

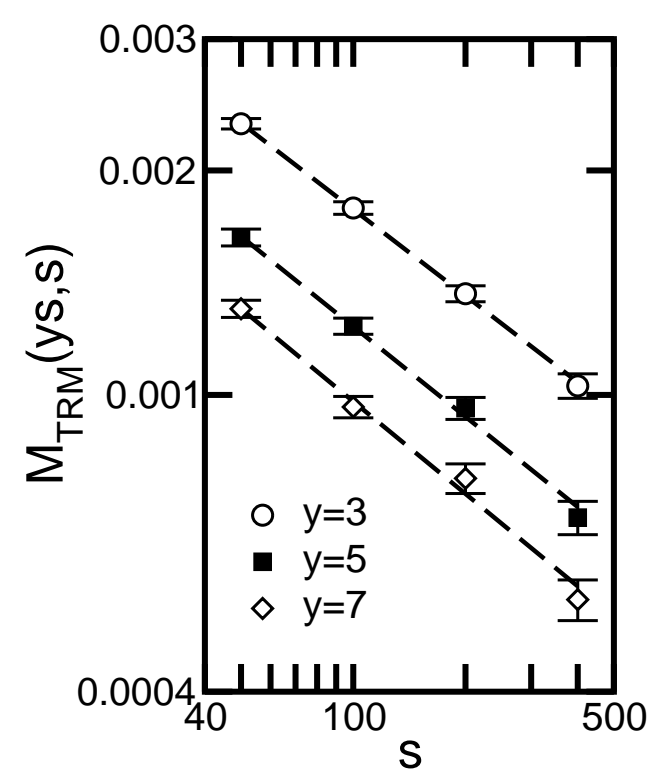

Fig. 4. Thermoremanent magnetization $M_{\mathrm{TRM}}(y s, s)$ of the two-dimensional random-bond Ising model (40) as a function of $s$ for $\varepsilon=0.5$ and $T=1.0$ and different values of $y$.

The random-bond Ising model is very well suited for investigating in detail the possible applicability of the theory of local scale-invariance to nontrivial systems undergoing phase-ordering. This is of course due to the fact that the dynamical exponent can be continuously changed by changing the distribution of the couplings and/or the temperature, yielding values of $z$ much larger than the value $z=2$ encountered in non-disordered ferromagnets undergoing phase-ordering. We have seen in section 2 for what models the data for $M_{\mathrm{TRM}}(t, s)$ agree with LSI, see table 2. Figure 5 compares for three different cases the computed scaling function 
Table 5. Quantities describing the dynamical scaling behaviour of the linear response $M_{T R M}$ in the bond-disordered two-dimensional Ising model for different values of $\epsilon$ and different temperatures $T$. The last column is obtained from equation (39) and should be compared with the numerical estimates for the exponent $a$.

\begin{tabular}{|rc|cl|cc|c|}
\hline \hline$\varepsilon$ & $T$ & $a$ & $\lambda_{R} / z$ & $r_{0}$ & $r_{1}$ & $1 / z$ \\
\hline \hline 0.5 & 1.0 & $0.398(5)$ & $0.61(1)$ & $0.021(1)$ & 0 & 0.400 \\
& 0.8 & $0.382(4)$ & $0.595(10)$ & $0.020(1)$ & 0 & 0.381 \\
& 0.6 & $0.353(4)$ & $0.58(1)$ & $0.022(1)$ & 0 & 0.353 \\
0.4 & $0.310(5)$ & $0.52(1)$ & $0.029(2)$ & $0.008(1)$ & 0.308 \\
\hline 1.0 & 1.0 & $0.330(5)$ & $0.51(1)$ & $0.021(1)$ & $-0.009(1)$ & 0.333 \\
& 0.8 & $0.308(4)$ & $0.49(1)$ & $0.019(1)$ & $-0.006(1)$ & 0.308 \\
& 0.6 & $0.277(6)$ & $0.46(1)$ & $0.020(1)$ & $-0.007(1)$ & 0.273 \\
0.4 & $0.22(1)$ & $0.375(10)$ & $0.026(2)$ & $-0.014(3)$ & 0.222 \\
\hline 2.0 & 1.0 & $0.24(2)$ & $0.33(1)$ & $0.048(2)$ & $-0.048(4)$ & 0.250 \\
0.8 & $0.22(2)$ & $0.30(1)$ & $0.093(3)$ & $-0.042(4)$ & 0.222 \\
0.6 & $0.17(2)$ & $0.27(1)$ & $0.194(4)$ & $-0.033(3)$ & 0.188 \\
\hline \hline
\end{tabular}

$f_{M}$ of the thermoremanent magnetization with the LSI prediction (43). The same comparison was made for $\varepsilon=0.5, T=1, \varepsilon=1, T=0.6$, and $\varepsilon=2, T=1$ in our original paper [66]. It has to be recalled that prior to this comparison the values of the exponents entering into (43) and also the normalization are already fixed. We see that the form of the scaling function (after subtraction of the above-mentioned finite-time correction, if needed) is perfectly described by LSI, and this is the case for all values of $\varepsilon$ and $T$ investigated. This is a very remarkable result as it suggests that the idea of extending dynamical scaling to local, space- and time-dependent, scaling is capable of reproducing faithfully the linear responses of very different systems characterized by very different values of the dynamical exponent.

Having looked at the evidence in favour of simple scaling of $M_{\mathrm{TRM}}(t, s)$, it may be useful here to take a slightly broader point of view. Indeed, in the discussion of glassy systems, people often describe the scaling of, say, the two-time correlation function as follows, e.g. [120]

$$
C(t, s)=C_{\text {st }}(t-s)+C_{\text {age }}(t, s)
$$

such that the stationary part satisfies $\lim _{t \rightarrow \infty} C_{\mathrm{st}}(t)=0$ and furthermore $\lim _{t-s \rightarrow \infty}\left(\lim _{s \rightarrow \infty} C_{\text {st }}(t-s)\right)=q_{\mathrm{EA}}$, where $q_{\mathrm{EA}}$ is the Edwards-Anderson orderparameter for glasses. On the other hand, the ageing part is assumed to read

$$
C_{\text {age }}(t, s)=\mathcal{C}\left(\frac{h(t)}{h(s)}\right), h(t)=h_{0} \exp \left[\frac{1}{A} \frac{t^{1-\mu}-1}{1-\mu}\right]
$$

where $\mathcal{C}$ is a scaling function, $\mu$ is a free parameter and $h_{0}$ and $A$ are constants. Conventionally, one refers to the case $0<\mu<1$ as subageing, the limit case $\mu \rightarrow 1$ as (full or simple) ageing and the case $\mu>1$ as superageing. However, it has been shown by Kurchan [83] that, given that the positive function $\mathcal{C}(u)$ decreases strictly 

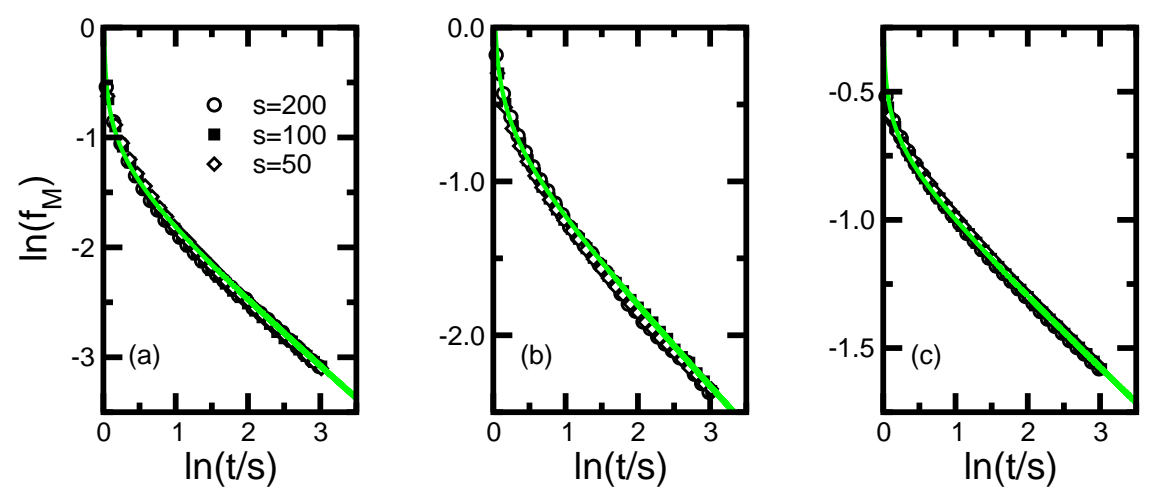

Fig. 5. Comparison of the numerically determined scaling functions $f_{M}(t / s)$ of the two-dimensional random-bond Ising model with the LSI prediction eq. (43) (full lines) for (a) $\varepsilon=0.5$ and $T=0.6$, (b) $\varepsilon=1$ and $T=1$ and (c) $\varepsilon=2$ and $T=0.6$.

monotonously with $u$, the case $\mu>1$ is incompatible with an elementary property of the autocorrelation function, namely that if there is a strong correlation between times $t_{1}$ and $t_{2}>t_{1}$ and as well a strong correlation between times $t_{2}$ and $t_{3}>t_{2}$, then there must exist a strong correlation between times $t_{1}$ and $t_{3}$. Hence superageing is impossible [83].

There is a nice argument which explains the origin of the form $(44,45)$ [5]. It relies on the observation that if $C(t, s)$ is plotted over against $t-s$, a plateau is observed, see also figure 2a. Further, according to Zippold, Kühn et Horner [122] the transition towards the scaling regime occurs at a time difference $t-s \sim t^{\zeta}$ where $0<\zeta<1$ describes this change of behaviour. ${ }^{7}$ Hence close to the plateau one can assume

$$
C(t, s)=q_{\mathrm{EA}}+t^{-\alpha} g_{1}\left((t-s) t^{-\zeta}\right)+\ldots
$$

where $\alpha$ is some exponent and $g_{1}$ a scaling function. Now, consider $t-s=x t^{\zeta}$ and take the limit $t \rightarrow \infty$ and then $x \gg 1$. Then, to leading order

$$
C(t, s)=\mathcal{C}\left(\frac{h(t)}{h(s)}\right) \simeq \mathcal{C}\left(1+x t^{\zeta} \frac{\mathrm{d} \ln h(t)}{\mathrm{d} t}\right) \simeq q_{\mathrm{EA}}+c_{\mathrm{age}}^{(1)}\left(x t^{\zeta} \frac{\mathrm{d} \ln h(t)}{\mathrm{d} t}\right)^{\mathfrak{b}}
$$

where $\mathfrak{b}$ is a further exponent which can be worked out for certain model spin glasses [5]. Comparing eqs. $(46,47)$, the dependences on $t$ and on $x$ can be separated which in particular leads to $\mathrm{d} \ln h(t) / \mathrm{d} t=A^{-1} t^{-\mu}$, where $\mu=\zeta+\alpha / \mathfrak{b}$ and $A$ is a separation constant. Then eq. (45) follows directly [5]. In particular, this means that other phenomenological forms which have been discussed in the past can be eliminated.

After these preparations we turn to a dicussion of ageing properties of the autocorrelation function in disordered ferromagnets. Indeed, recent studies of $C(t, s)$ in bond-diluted [66] and in site-diluted [99] Ising models quenched to below the critical points showed surprising and at first sight unexpected features. In figure $6 \mathrm{a}$ we show data [66] for the two-dimensional random-bond Ising model plotted over against $t / s$.

\footnotetext{
${ }^{7}$ Explicitly, $\zeta=4 /(d+2)$ in the $d$-dimensional spherical model and $\zeta=4 / 5$ in the spherical spin glass $[122]$.
} 

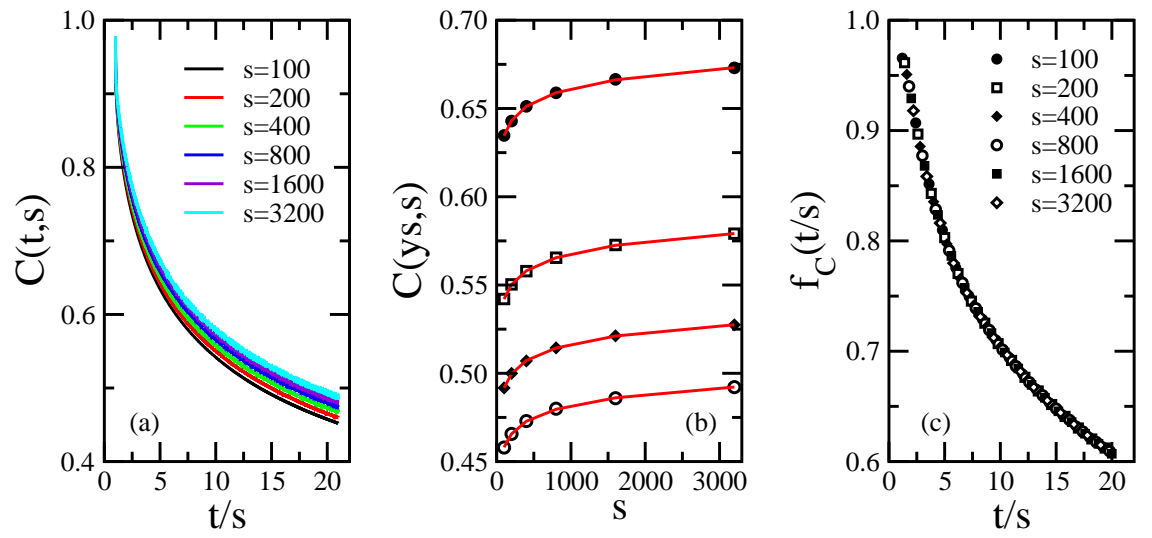

Fig. 6. (a) Autocorrelation $C(t, s)$ vs $t / s$ for various waiting times $s$ in the randombond Ising model with $\varepsilon=2$ and $T=1$. (b) Plot of $C(y s, s)$ for the same parameters and $y=5,10,15,20$ (from top to bottom). The data can be perfectly fitted by the scaling form (48) with $b^{\prime}=0.075$, which illustrates the existence of important finitetime corrections. (c) The scaling function $f_{C}(t / s)$ obtained after subtracting off the finite-time corrections from the autocorrelation, again for $\varepsilon=2$ and $T=1$, in agreement with simple ageing of the autocorrelation.

In clear contrast with non-disordered simple magnets, no clear scaling is seen. At first sight, one might be tempted to introduce a non-vanishing ageing exponent $b$, see eq. (5), but since the curves for $C(t, s)$ increase with $s$, the effective values of $b=b_{\text {eff }}$ fitted to the data will turn out to be negative $[66,99]$. If true, that would imply that for $s \rightarrow \infty$ the autocorrelator $C(t, s)$ would grow unboundedly, which is impossible. It is therefore necessary to reexamine whether the standard scaling form of simple ageing is applicable. Indeed, in a recent study of the randomly site diluted Ising model [99] the more general scaling form $(44,45)$ was used and fitted values of $\mu$ in the range $\mu \approx 1.03-1.04$ were reported, implying the so-called superageing behaviour [99]. Although a good collapse of the data can be obtained this way, the arguments raised by Kurchan [83] and quoted above make it doubtful that the conclusion of [99] can be accepted at face value. Indeed, in using scaling forms such as (5) one has to be careful about the possibility of finite-time corrections to scaling. We illustrate the presence of strong corrections to scaling in figure $6 \mathrm{~b}[68]$ for the case $\varepsilon=2$ and $T=1$, for several values of $y=t / s$. Clearly, the data are perfectly described by the extended scaling form

$$
C(y s, s)=f_{C}(y)-s^{-b^{\prime}} g_{C}(y)
$$

(hence with $b=0$ ) and with $b^{\prime}>0 .{ }^{8}$ Subtracting off the leading correction term, yields a perfect scaling behaviour eq. (5) according to simple ageing as shown in figure 6c [68], quite analogous with the standard scaling expected for simple magnets quenched to below their critical point.

\footnotetext{
${ }^{8}$ For some other values of $\varepsilon$ and of $T$ two correction terms have to be included in order to describe the non-monotonic variation of $C(y s, s)$ as a function of $s$, see [68] for details.
} 
In conclusion, the random-bond Ising model apparently ages in much the same way as a simple magnet. The prediction of table 1 for the exponents $a, b$ in class $\mathrm{S}$ systems hold true for this non-glassy disordered system as well, but the value of the dynamical exponent $z=z(T, \varepsilon)$ (and also of $\lambda_{C, R}$ ) becomes dependent on temperature and on the distribution of the couplings. The form of the response function agrees with the prediction of LSI, for a large range of values of $z$. It would be very interesting to check these conclusions in other systems.

\section{Critical Ising spin glasses}

The disordered ferromagnet studied in the previous section can be viewed as intermediary between the simple, non-disordered magnets and the disordered and highly frustrated spin glasses. In the following we discuss to what extend the results obtained for the disordered ferromagnets can be extended to glassy systems. We thereby shall concentrate on the Ising spin glass, with a static Hamiltonian $\mathcal{H}=-\sum_{(i, j)} J_{i, j} \sigma_{i} \sigma_{j}$. Here $\sigma_{i}= \pm 1$ are the usual Ising spins and the nearestneighbour couplings $J_{i, j}$ are random variables. We shall consider three different distributions of the couplings: (i) the bimodal distribution with

$$
P_{B}\left(J_{i, j}\right)=\left[\delta\left(J_{i, j}-J\right)+\delta\left(J_{i, j}+J\right)\right] / 2,
$$

(ii) the Gaussian distribution with

$$
P_{G}\left(J_{i, j}\right)=\exp \left(-J_{i, j}^{2} / 2 J^{2}\right) /(J \sqrt{2 \pi})
$$

and (iii) the Laplacian distribution with

$$
P_{L}\left(J_{i, j}\right)=\exp \left(-\sqrt{2}\left|J_{i, j} / J\right|\right) /(J \sqrt{2}) .
$$

All distributions are symmetric with zero mean and variance $\left\langle J_{i, j}^{2}\right\rangle / J^{2}=1$. The dynamics of the model is given by a master equation where the rates are chosen according to heat-bath dynamics. It is by now established, see e.g. [82] for a review, that for $d>2$ dimensions this model undergoes an equilibrium phase-transition between a paramagnetic and a frustrated spin-glass phase. There has been considerable debate on the precise relationship between the relevant time and length scales for quenches below the spin-glass critical temperature $T_{c}$. It has been attempted to summarize the present state of knowledge into the form [19]

$$
t(L) \sim L^{z} \exp \left(\frac{\Delta_{0}}{T}\left(\frac{L}{\xi(T)}\right)^{\psi}\right)
$$

where $\Delta_{0}$ is an energy scale of order $T_{c}, \psi$ is a barrier exponent and $\xi(T)$ is the equilibrium correlation length at temperature $T$. This form has been used to fit successfully simulational data in the three-dimensional and in the four-dimensional Edwards-Anderson model [19, 18]. Although the typical length scales are merely of the order of a few lattice sizes, see e.g. [121], the relaxation times are sufficiently large for a dynamical scaling to set in. While the expression (52), if correct, points towards a cross-over behaviour between a simple power-law scaling and an exponential scaling for $T<T_{c}$ in spin glasses as would follow from the droplet model, it also suggests 

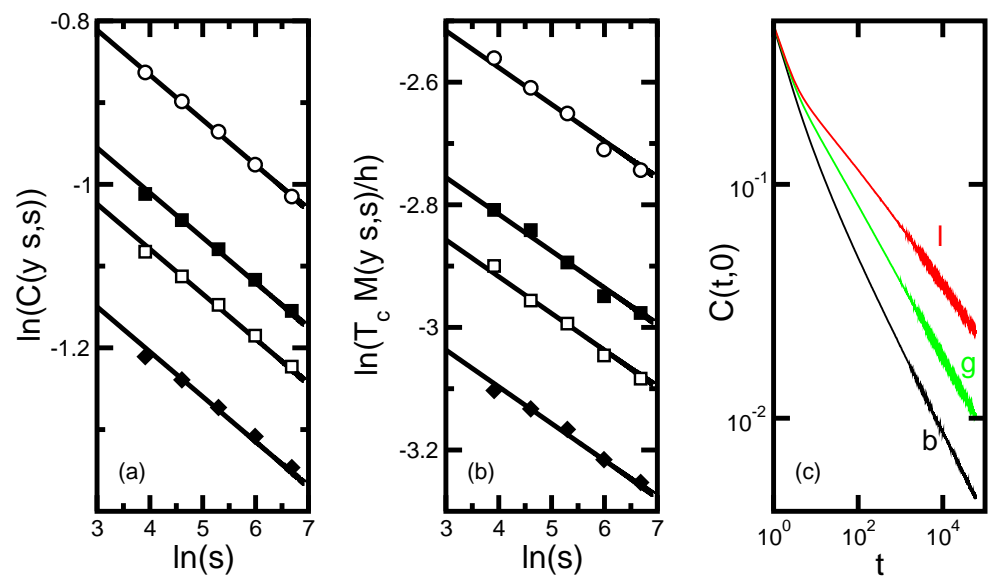

Fig. 7. Scaling of (a) the autocorrelation function $C(y s, s)$ and (b) the thermoremanent magnetization $M(y s, s)$ of the critical three-dimensional Edwards-Anderson spin glass with a bimodal distribution of the couplings for (from top to bottom) $y=5,8,10,15$. The full curves are $C(y s, s)=c_{0} s^{-b}$ and $M(y s, s)=m_{0} s^{-a}$, where $c_{0}, m_{0}$ were fitted to the numerical data. (c) Temporal evolution of the autocorrelation $C(t, 0)$ in the three-dimensional cases (b: bimodal, g: Gaussian, l: Laplacian).

that at criticality simple power-law scaling (in the sense discussed above in section 3) should prevail.

From now on we consider the three- and the four-dimensional Ising spin glass quenched to $T=T_{c}$ from a fully disordered initial state and discuss the dynamical scaling behaviour of critical two-time quantities. We consider two classes of observables: (a) thermoremanent magnetization where $h$ is constant up to a single jump and (b) alternating susceptibility, where $h=h(t)$ is oscillatory.

\subsection{Thermoremanent magnetization}

Let us first verify that the standard scaling forms (5) and (6) for the autocorrelation and the autoresponse are indeed observed in critical spin glasses. From the data shown in figure 7 for the case of a bimodal distribution of the couplings in three dimensions we conclude that both the correlation (figure 7a) and integrated response (figure $7 \mathrm{~b}$ ) are consistent with a power-law scaling. Similar results are obtained $[63,105]$ for the two other distributions, both in three and four space dimensions.

From the slopes of figure 7 we obtain the estimates for the exponents $a$ and $b$ gathered in table 6 . Interestingly, we observe that $a=b$ within our numerical errors for a fixed choice of the distribution of the couplings, but that the results for different distributions are different. Even more intriguingly, other critical nonequilibrium dynamical quantities, as for example the exponent $\lambda_{C} / z$ describing the decay of the correlations in the long-time limit or the limit value of the fluctuationdissipation ratio (8), also vary strongly and systematically with the form of the interaction distribution, see table 6 and figure 7c [105]. Here we emphasize the 
Table 6. Nonequilibrium quantities of the critical Ising spin glass for bimodal, Gaussian, and Laplacian distributions of the nearest-neighbour couplings in $d=3$ and $d=4$ dimensions.

\begin{tabular}{|c|cccc|}
\hline & \multicolumn{4}{|c|}{ bimodal } \\
\hline$d$ & $a$ & $b$ & $\lambda_{C} / z$ & $X_{\infty}$ \\
\hline 3 & $0.060(4)$ & $0.056(3)$ & $0.362(5)$ & $0.12(1)$ \\
4 & $0.18(1)$ & $0.180(5)$ & $0.615(10)$ & $0.20(1)$ \\
\hline & \multicolumn{4}{|c|}{ Gaussian } \\
\hline$d$ & $a$ & $b$ & $\lambda_{C} / z$ & $X_{\infty}$ \\
\hline 3 & $0.044(1)$ & $0.043(1)$ & $0.320(5)$ & $0.09(1)$ \\
4 & $0.169(4)$ & $0.171(2)$ & $0.58(1)$ & $0.175(10)$ \\
\hline & \multicolumn{4}{|c|}{ Laplacian } \\
\hline$d$ & $a$ & $b$ & $\lambda_{C} / z$ & $X_{\infty}$ \\
\hline 3 & $0.033(3)$ & $0.032(2)$ & $0.259(2)$ & $0.055(2)$ \\
4 & $0.143(5)$ & $0.140(3)$ & $0.54(1)$ & $0.13(1)$ \\
\hline
\end{tabular}

diagnostic usefulness of the universal limit fluctuation-dissipation ratio $X_{\infty}$ whose numerical estimates vary up to a factor of 2 between the various distributions. Having excluded the most probable sources of systematic errors [105], we interpret the numerical data as strong evidence that in spin glasses critical out-of-equilibrium quantities do depend on the exact form of the distribution of the couplings. ${ }^{9}$ It is not yet clear whether this intriguing property is only a purely dynamical effect. Whereas in the past some studies of static critical quantities observed a similar dependence on the distribution of the couplings [15], recent studies yielded for the different distributions values which agree within error bars [79, 81].

Let us now turn to the scaling functions themselves. Plotting the rescaled autocorrelator $s^{b} C(t, s)$ versus the scaling variable $t / s$ yields a nice data collapse compatible with a simple power-law scaling $L(t) \sim t^{1 / z}[63,105]$. In figure 8 we show the two-time scaling of the integrated response where again a collapse of the data in terms of a simple power-law scaling is observed. The fact that both the autocorrelator and the thermoremanent magnetization can be described in terms of such a power-law scaling is evidence in favour of the time-dependent length-scale (52). In principle, one would like to extract an exponent $\lambda_{R}^{\prime} / z$ from the slopes of the thermoremanent magnetization. It turns out that the values of $\lambda_{R}^{\prime} / z$ thus obtained are significantly different from the ones found for $\lambda_{C} / z$. For example, for the Ising spin glass with a bimodal distribution of the couplings one finds $\lambda_{R}^{\prime} / z=0.45$ (resp. 0.72) in three resp. four dimensions. On the other hand, since $X_{\infty}$ is finite, one must have $\lambda_{R}=\lambda_{C}$, but it may be necessary to go to very large values of $y=t / s$ in order to see this. Indeed, if one considers larger values of $y=t / s$ as is shown in the insets of figure $8 \mathrm{ab}$, one observes a passage from an effective exponent $\lambda_{R}^{\prime} / z$ at intermediate values of the scaling variable $y$ to the truly asymptotic value $\lambda_{R} / z$ at larger values of $y$. Because of this passage from an effective exponent $\lambda_{R}^{\prime} / z$ at intermediate values

\footnotetext{
${ }^{9}$ We recall that a similar result was already seen for the disordered, but unfrustrated, Ising model in section 3.
} 

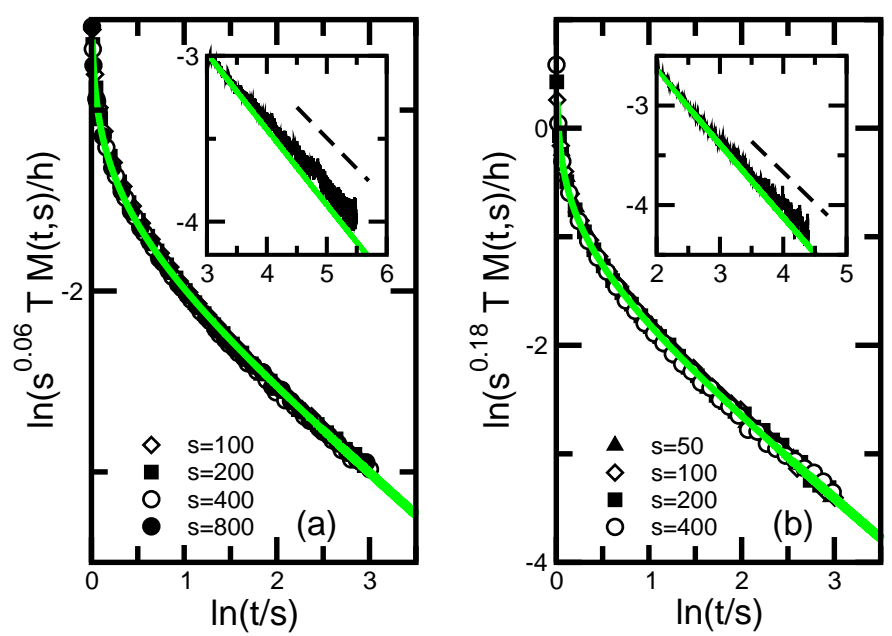

Fig. 8. Scaling of the thermoremanent magnetization in the Edwards-Anderson spin glass at criticality with a bimodal distribution of the couplings in (a) three and (b) four dimensions. The full curve is the prediction (43) of local scale-invariance. The results of very longs runs for a single waiting time are shown in the insets where (a) $s=100$ in three dimenions and (b) $s=25$ in four dimensions.

of $y=t / s$ to the truly asymptotic value for larger $y$ we cannot expect the LSI equation (43) to describe the scaling function $f_{M}(y)$ for all values of $y=t / s$. In figure $8 \mathrm{ab}$ we compare the numerical data, in both three and four dimensions with the prediction (43) where we have inserted the values of the exponents $a$ and $\lambda_{R}^{\prime} / z$. We find a nice agreement of the prediction (43) of local scale-invariance with our data for $y=t / s$ not too large, but for very large arguments the precise behaviour of the scaling function $f_{M}(y)$ cannot be fully reproduced.

\subsection{Alternating susceptibility}

An interesting possibility to further test the behaviour of critical spin-glass models consists of working with a time-dependent (oscillating) magnetic field and to study simultaneously the dependence on time and on the imposed oscillation angular frequency $\omega$. For a harmonic magnetic field, it is common to consider the real and the imaginary part of the magnetic susceptibility

$$
\begin{aligned}
& \chi^{\prime}(\omega, t)=\int_{0}^{t} \mathrm{~d} u R(t, u) \cos (\omega(t-u)) \\
& \chi^{\prime \prime}(\omega, t)=\int_{0}^{t} \mathrm{~d} u R(t, u) \sin (\omega(t-u))
\end{aligned}
$$

where $R(t, s)$ is the linear response discussed above. In this setting, $1 / \omega$ provides the second time-scale, the natural scaling variable is $y=\omega t$ and the scaling regime should be reached in the limit $\omega \rightarrow 0$ and $t \rightarrow \infty$. In many experiments and simulations, one averages over at least one period of the oscillating field, see e.g. [101]. 
Then in a great variety of glass-forming substances quenched to below or near to their glass transition point one observes good but not always perfect evidence for an $\omega t$-scaling behaviour of the following form of the period-averaged dissipative (imaginary) part [78, 117]

$$
\overline{\chi^{\prime \prime}}(\omega, t)=\chi_{\text {st }}^{\prime \prime}(\omega)+\chi_{\text {age }}^{\prime \prime}(\omega, t), \quad \chi_{\text {age }}^{\prime \prime}(\omega, t) \simeq A_{\text {age }}^{\prime \prime}(\omega t)^{-b^{\prime \prime}}
$$

where $\chi_{\text {st }}^{\prime \prime}$ is thought of as a 'stationary' contribution while the ageing behaviour is described by $\chi_{\text {age }}^{\prime \prime}$. The amplitude $A_{\text {age }}^{\prime \prime}$ and the exponent $b^{\prime \prime}$ are obtained from fits to the experimental data. Similar scaling forms have been proposed for the dispersive (real) part $\overline{\chi^{\prime}}$ but in practice the imaginary part is usually easier to measure. It is usually thought that $b^{\prime}=b^{\prime \prime}$.

Assuming the validity of LSI, we have derived the scaling relation [62]

$$
b^{\prime}=b^{\prime \prime}=a-a^{\prime}
$$

where $a^{\prime}$ is the exponent defined in eq. (34). Hence this method gives direct access to the exponent $a-a^{\prime}$ and should be useful for future direct tests of whether $a$ and $a^{\prime}$ are really different.

In order to derive (55), we notice the central rôle played by the time difference $\tau=t-u$ in (54) [56]. Depending on its value either an equilibrium behaviour or else an ageing behaviour is obtained. Recall from section 3 that there is a time-scale $t_{p} \sim t^{\zeta}$ with $0<\zeta<1$ on which the transition between the two regimes occurs [122] such that $R(t, s) \simeq R_{\text {eq }}(t-s)$ for $t-s \lesssim t_{p}$ and $R(t, s)=s^{-1-a} f_{R}(t / s)$ as given in eq. (34) for $t-s \gtrsim t_{p}$. In addition, one measures for $u \approx t$ the response with respect to a change in the initial conditions and then instead of (34) one expects $R \approx R_{\text {ini }}(t) \sim t^{-\lambda_{R} / z}[20] .{ }^{10}$. We therefore must introduce a further time-scale $t_{\varepsilon}$ such that $t-t_{\varepsilon}=\mathrm{O}(1)$. Splitting the integral into three terms corresponding to these three regimes, one has

$$
\begin{aligned}
\chi^{\prime \prime}(\omega, t)=\int_{0}^{t} \mathrm{~d} \tau R(t, t-\tau) \sin \omega \tau \\
=\int_{0}^{t_{p}} \mathrm{~d} \tau R(t, t-\tau) \sin \omega \tau+\int_{t_{p}}^{t_{\varepsilon}} \mathrm{d} \tau R(t, t-\tau) \sin \omega \tau \\
\quad+\int_{t_{\varepsilon}}^{t} \mathrm{~d} \tau R(t, t-\tau) \sin \omega \tau \\
\simeq \int_{0}^{t_{p}} \mathrm{~d} \tau R_{\mathrm{eq}}(\tau) \sin \omega \tau+t^{-a} \int_{t_{p} / t}^{t_{\varepsilon} / t} \mathrm{~d} v f_{R}\left(\frac{1}{1-v}\right) \frac{\sin \omega t v}{(1-v)^{1+a}} \\
\quad+t^{-\lambda_{R} / z} \int_{t_{\varepsilon}}^{t} \mathrm{~d} \tau c_{0} \sin \omega \tau \\
=\chi_{1}^{\prime \prime}(\omega)+t^{-a} \int_{0}^{1} \mathrm{~d} v f_{R}\left(\frac{1}{1-v}\right) \frac{\sin \omega t v}{(1-v)^{1+a}}+\mathrm{O}\left(t^{-\lambda_{R} / z}\right) \\
=\chi_{1}^{\prime \prime}(\omega)+t^{-a} \chi_{2}^{\prime \prime}(\omega t)+\mathrm{O}\left(t^{-\lambda_{R} / z}\right)
\end{aligned}
$$

In the third line, we used the asymptotic forms of $R(t, s)$ as described above. This means that the cross-over between the equilibrium and the ageing regimes is assumed

${ }^{10}$ A similar argument is used in the derivation of eqs. $(42,43)[56,60]$ 
to be very rapid. While this is certainly correct for simple magnets, its validity in spin-glasses is far from obvious. In the last line, we restricted ourselves to the longtime limit $t \rightarrow \infty$. Here, the function $\chi_{1}^{\prime \prime}(\omega)$ merely depends on the equilibrium form of the linear response $R_{\text {eq }}(t, s)$. It also becomes clear that the often-found stationary term in the integrated response [29, 31, 34, 40, 78, 82, 108, 109, 117] does not necessarily require the separation of a similar 'stationary' part in the response function $R(t, s)$ itself. An analogous expression can be derived for $\chi^{\prime}(\omega, t)$.

We now insert the prediction (34) of LSI. Then the scaling functions read, together with their leading behaviour as $y \rightarrow \infty[62]$

$$
\begin{aligned}
\chi_{2}^{\prime \prime}(y)= & f_{0} B\left(1-a^{\prime}, \frac{\lambda_{R}}{z}-a\right) y^{1-a} \\
& \times_{2} F_{3}\left(\frac{1-a^{\prime}}{2}, \frac{2-a^{\prime}}{2} ; \frac{3}{2}, \frac{1-a-a^{\prime}}{2}+\frac{\lambda_{R}}{2 z}, \frac{2-a-a^{\prime}}{2}+\frac{\lambda_{R}}{2 z} ;-\frac{y^{2}}{4}\right)(57) \\
\simeq & f_{1}^{\prime \prime} y^{a^{\prime}-a}+f_{2}^{\prime \prime} y^{-\lambda_{R} / z} \sin \left(y+\frac{\pi}{2}\left[a-\lambda_{R} / z\right]\right) \\
\chi_{2}^{\prime}(y)= & f_{0} B\left(-a^{\prime}, \frac{\lambda_{R}}{z}-a\right) y^{-a} \\
& \times_{2} F_{3}\left(\frac{-a^{\prime}}{2}, \frac{1-a^{\prime}}{2} ; \frac{1}{2}, \frac{-a-a^{\prime}}{2}+\frac{\lambda_{R}}{2 z}, \frac{1-a-a^{\prime}}{2}+\frac{\lambda_{R}}{2 z} ;-\frac{y^{2}}{4}\right) \\
\simeq & f_{1}^{\prime} y^{a^{\prime}-a}+f_{2}^{\prime} y^{-\lambda_{R} / z} \cos \left(y+\frac{\pi}{2}\left[a-\lambda_{R} / z\right]\right)
\end{aligned}
$$

where ${ }_{2} F_{3}$ is a hypergeometric function and $f_{1,2}^{\prime}$ and $f_{1,2}^{\prime \prime}$ are known constants proportional to the normalization constant $f_{0}$. We see that there appear terms which decrease monotonously with $y$ but that there are also oscillating terms. They are described by different exponents and must be extracted by a different experimental setup. The oscillating terms follow the oscillations of the external field and the decrease of the oscillation amplitude gives a direct access to the exponent $\lambda_{R} / z$. On the other hand, in many experiments the data are averaged over one or several periods of the external field. For $y$ sufficiently large, the contribution of the oscillating term in eqs. $(57,58)$ vanishes after averaging and then only a simple algebraic component remains, which permits to extract the exponent $a-a^{\prime}$. For period-averaged data or else if $\lambda_{R} / z \geq a-a^{\prime}$, the leading behaviour for large arguments is

$$
\chi_{2}^{\prime}(y) \sim \chi_{2}^{\prime \prime}(y) \sim y^{a^{\prime}-a}
$$

and the scaling relations (55) follow. Since the new exponent $a^{\prime}$ enters in (55), this suggests that no scaling relation between $b^{\prime}=b^{\prime \prime}$ and the other ageing exponents, with $a^{\prime}$ excluded, exists.

We now describe a test [62] of the predictions eqs. $(57,58)$ in the threedimensional Ising spin glass with bimodal disorder, quenched to its critical point $T_{c} \approx 1.19$. In order to study the alternating susceptibility far from equilibrium we prepared the system in an uncorrelated initial state (corresponding to an infinite initial temperature) before quenching it to the critical point at time $t=0$. At the same time an external oscillating magnetic field

$$
h(t)=h_{0} \cos \omega t
$$

was switched on, with its amplitude fixed at $h_{0}=0.05$ which is well inside the linearresponse regime. We consider different values of the angular frequency $\omega=2 \pi / p$ 
with $p$ ranging from 50 to 1600 . Numerically, the in-phase and the out-of-phase susceptibilities are given by the expressions [4]

$$
\chi^{\prime \prime}(\omega, t)=m(t) \sin \omega t, \quad \chi^{\prime}(\omega, t)=m(t) \cos \omega t
$$

with $m(t)=\sum_{i} \sigma_{i}(t)$ but the equilibrium parts of $\chi^{\prime \prime}$ and $\chi^{\prime}$ must be subtracted off $[62]$.

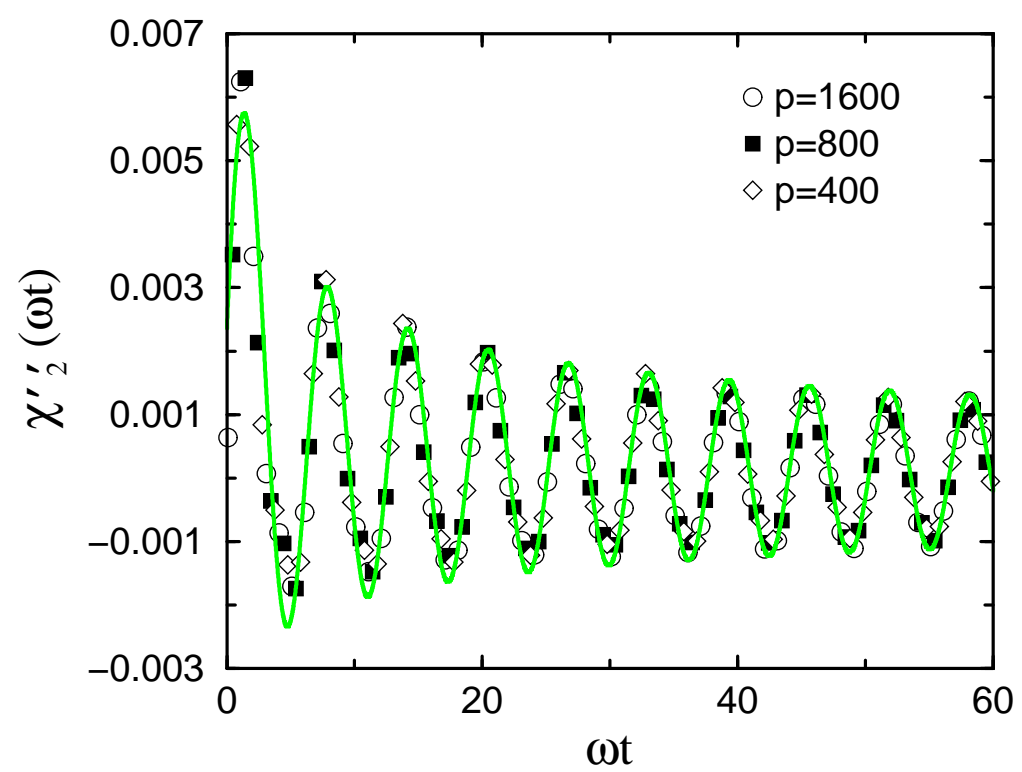

Fig. 9. Scaling of the dissipative part $\chi_{2}^{\prime \prime}(\omega t)$ of the alternating susceptibility as function of the scaling variable $\omega t$ for different angular frequencies $\omega=2 \pi / p$ with $p=1600,800$, and 400. The full curve is the theoretical prediction (57) with $f_{0}=$ 0.002 and $a^{\prime}=-0.70$ but which has also been shifted horizontally by $y \rightarrow y+\Delta y$, with $\Delta y=-0.45$, see text. Statistical error bars are smaller than the symbol sizes.

In figures 9 and 10 [62] we compare the expected scaling behaviour of the ageing part

$$
\chi_{2}^{\prime \prime}=\chi_{2}^{\prime \prime}(\omega t) \quad \text { and } \quad \chi_{2}^{\prime}=\chi_{2}^{\prime}(\omega t) .
$$

For the larger values of $p$, corresponding to the smaller values of $\omega$, one observes a very good data collapse for both quantities, which furnishes clear evidence in favour of a power-law scaling at $T=T_{c}$. The value $a=0.060(4)$ determined previously from the decay of the thermoremanent magnetization $[63,105]$ was used. For smaller values of $p$, the collapse is less good. It is possible that for the corresponding values of $\omega$ the dynamical scaling regime is not yet reached.

The data shown in these two figures can in principle be compared directly with the analytical predictions (57) and (58), provided however, that the positions of maxima of $\chi_{2}^{\prime \prime}$ and $\chi_{2}^{\prime}$ are shifted by the amount $\Delta y \approx-0.45$ when compared with 


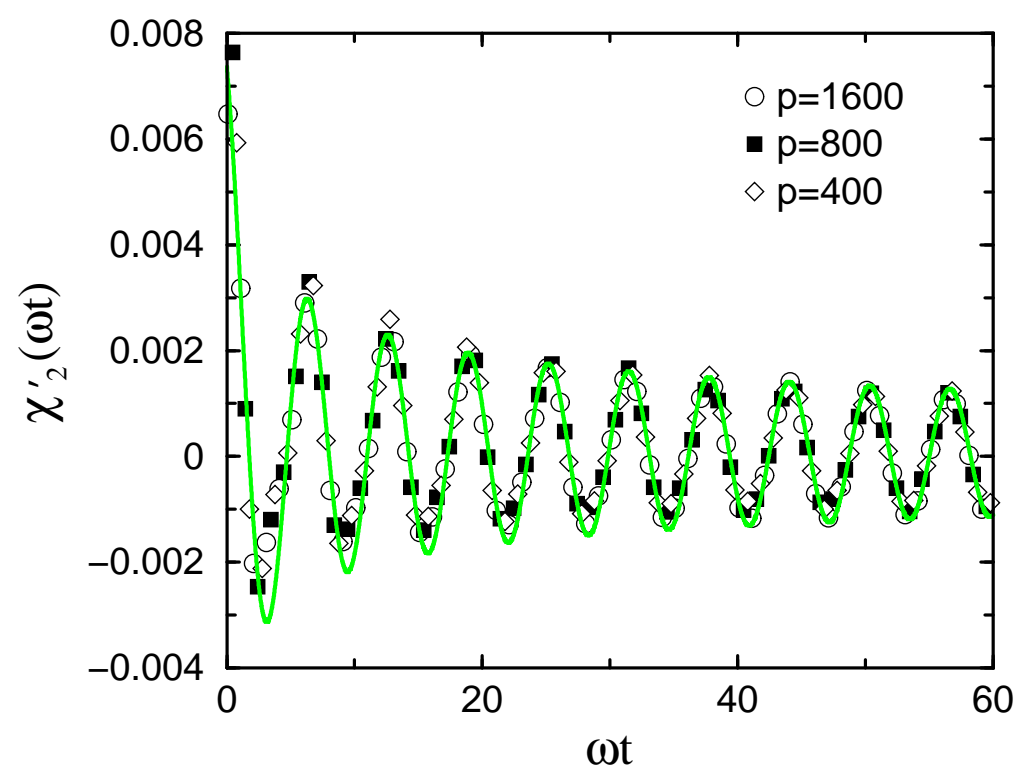

Fig. 10. The same as in Figure 9, but now for the dispersive part $\chi_{2}^{\prime}(\omega t)$. The full curve is the shifted theoretical prediction (58) with $f_{0}=0.002$ and $a^{\prime}=-0.70$.

the positions obtained in the analytical treatment of the previous section. Where this shift comes from is not understood. It is possible, however, that the crossover between the equilibrium and the ageing regimes is not almost instantaneous in contrast to what we assumed in the derivation of eq. (56).

Since both $a$ and $\lambda_{R} / z$ were known before $[63,105]$ the only free parameters in this comparison are the amplitude $f_{0}$ and the exponent $a^{\prime}$. The final estimates are $[62]$

$$
a^{\prime}=-0.70(3), f_{0}=0.00203(1)
$$

and the fit is compared to the data in the figures. These values (63) of the parameters describe consistently both $\chi_{2}^{\prime \prime}$ and $\chi_{2}^{\prime}$. Although some discrepancies are observed for small values of $y=\omega t$, the overall agreement between the simulation and the shifted theoretical prediction is very good. Clearly, LSI captures an essential part of the ageing behaviour of critical spin glass models.

We finish with a discussion of existing experimental results on the scaling of the alternating susceptibility. A detailed discussion of a possible scaling of the alternating susceptibility was presented by Suzuki and Suzuki for the short-ranged Ising spin glass $\mathrm{Cu}_{0.5} \mathrm{Co}_{0.5} \mathrm{Cl}_{2}-\mathrm{FeCl}_{3}$ graphite bi-intercalation compound [117]. After a rapid quench to below the glass temperature $T_{g}=3.92(11) \mathrm{K}$, they measure $\chi^{\prime \prime}(\omega, t)$ for fixed $\omega$ and find that their (period-averaged) data are well-fitted by the power-law

$$
\overline{\chi^{\prime \prime}}(\omega, t)=\chi_{0}^{\prime \prime}(\omega)+A^{\prime \prime}(\omega) t^{-b^{\prime \prime}}
$$

While the fitted exponent $b^{\prime \prime}$ depends only slightly on $\omega$, they further show evidence for a power-law $A^{\prime \prime}(\omega)=A_{0}^{\prime \prime} \omega^{-\mu^{\prime \prime}}$ and find that "...the value of $\mu^{\prime \prime}$ is almost the 
same as that of $b^{\prime \prime} "[117]$. In this way, they arrive at the $\omega t$-scaling form

$$
\overline{\chi^{\prime \prime}}(\omega, t)=\chi_{0}^{\prime \prime}(\omega)+A_{0}^{\prime \prime}(\omega t)^{-b^{\prime \prime}}
$$

and a similar form for $\overline{\chi^{\prime}}(\omega, t)$ where "..$b^{\prime}$ and $b^{\prime \prime}$ are of the same order at the same temperature" [117]. Experimentally measured values of the exponent $b^{\prime \prime}$ (and also $b^{\prime}$ ) of some materials are collected in table 7 . Comparing the experimental results of Suzuki and Suzuki [117] with the theoretical scaling form (56), one sees:

1. the experimental evidence for a pure $\omega t$-scaling indicates that the exponent $a$ must indeed be very small.

2. when considering the leading behaviour for $y=\omega t$ large (their data go up to $\left.y \lesssim 10^{6}[117]\right)$ and recalling that the experimental data are averaged over at least one period of the external field, one can read off

$$
b^{\prime \prime}=a-a^{\prime}, \mu^{\prime \prime}=-a^{\prime}
$$

and the observed [117] near equality $b^{\prime \prime} \approx \mu^{\prime \prime}$ is again consistent with $a$ being negligibly small.

3. the data are consistent with the theoretically requested relation $b^{\prime}=b^{\prime \prime}$.

Table 7. Measured values of the exponents $b^{\prime \prime}$ and $b^{\prime}$ in several glassy materials, using the scaling form (54). Here $T_{g}$ stands for the glass transition temperature and $T$ is the temperature where the data were taken. For $\mathrm{Fe}_{0.5} \mathrm{Mn}_{0.5} \mathrm{TiO}_{3}$ and $\mathrm{CdCr}_{1.7} \mathrm{In}_{0.3} \mathrm{~S}_{4}$ the relation $b^{\prime}=b^{\prime \prime}$ was assumed. The simulational results in the critical Ising spin

\begin{tabular}{|c|c|c|c|c|c|c|}
\hline Material & $\bar{T} T_{g}[\mathrm{~K}]$ & $\bar{T}[\mathrm{~K}]$ & $\overline{b^{\prime \prime}}$ & $\overline{b^{\prime}}$ & Ref. & \\
\hline $\mathrm{Cu}_{0.5} \mathrm{Co}_{0.5} \mathrm{Cl}_{2}-\mathrm{FeCl}_{3}$ & $3.92(11)$ & 3.25 & $0.01(4)$ & $\overline{0.08(3)}$ & {$[117]$} & Ising \\
\hline - GBIC & & 3.5 & $0.017(32)$ & $0.05(2)$ & & spin glass \\
\hline & & 3.75 & $0.16(3)$ & $0.20(2)$ & & \\
\hline & & 3.85 & $0.15(3)$ & & & \\
\hline & & 3.95 & $0.16(4)$ & $0.20(2)$ & & \\
\hline $\mathrm{Fe}_{0.5} \mathrm{Mn}_{0.5} \mathrm{TiO}_{3}$ & 20.7 & 15 & $0.14(3)$ & & {$[39,37]$} & Ising \\
\hline & & 19 & $0.14(3)$ & & & spin glass \\
\hline $\mathrm{CdCr}_{1.7} \operatorname{In}_{0.3} \mathrm{~S}_{4}$ & 16.7 & 12 & $0.18(3)$ & & {$[39,37]$} & Heisenberg \\
\hline & & 14 & $0.18(3)$ & & & spin glass \\
\hline $\mathrm{CdCr}_{2 x} \operatorname{In}_{2-2 x} \mathrm{~S}_{4} x=0.95$ & 70 & 8 & 0.2 & & {$[38,37]$} & disordered \\
\hline & & 67 & 0.2 & & & ferromagnet \\
\hline$x=0.90$ & 50 & 42 & 0.20 & & {$[119,37]$} & \\
\hline $\mathrm{Pb}\left(\mathrm{Mg}_{1 / 3} \mathrm{Nb}_{2 / 3}\right) \mathrm{O}_{3}$ & $\sim 220$ & $\lesssim 220$ & 0.17 & & {$[31]$} & $\begin{array}{l}\text { relaxor } \\
\text { ferroelectric }\end{array}$ \\
\hline$\left(T=T_{c}\right)$ & & & $0.76(3)$ & $\overline{0.76(3)}$ & [62] & $\begin{array}{l}\text { Ising } \\
\text { spin glass }\end{array}$ \\
\hline
\end{tabular}
glass are also included.

Similar values of $b^{\prime \prime}$ were observed for several other materials, quite independently of the precise physical nature as can be seen from table 7 , but the errors are still too large to permit a discussion of the universality of the exponents. However, 
the experimental data are in many of these systems at least as well described by a logarithmic scaling as expected from the droplet theory [37, 78, 39]. Furthermore, in several systems also strong deviations from a simple $\omega t$-scaling were found, see [32]. Finally, we mention that in systems like $\beta$-hydroquinol-clathrate [108] or even simple liquids like glycerol [85] a power-law dependence of the form $\chi_{\text {age }}^{\prime \prime} \sim t^{-a}$ or $\chi_{\text {age }}^{\prime} \sim t^{-a}$ was observed. All in all, it is at present not completely clear why one should find a simple $\omega t$-scaling of the alternating susceptibility whereas subageing is frequenlt admitted for the thermoremanent magnetization.

A last important observation follows from comparing the experimental data for the exponents $b^{\prime}=b^{\prime \prime} \approx 0.1-0.2$ for all the $3 D$ materials studied so far with the theoretical estimate $b^{\prime \prime} \simeq 0.76$ derived from the $3 D$ Ising spin glass with binary disorder. The values are very far from each other and this huge difference calls for an explanation. Could this be seen as an indication that the spin glass models considered by theorists only capture imperfectly what is going on in real materials ?

\section{Discussion}

The available evidence for universality in the ageing behaviour seen in either glassy materials, disordered systems or else simple many-body systems without disorder may be taken, in combination with dynamical scaling, as suggestive of the existence of deeper dynamical symmetries in such systems. We have advocated here the point of view that such a new symmetry might be sought through an extension of standard dynamical scaling towards a local scale-invariance. The present formulation of the theory has been built around dynamical scaling as it occurs in full ageing, but this was mainly for reasons of technical simplicity and one could consider different forms of dynamical scaling, with a modified form of the Lie algebra generator $X_{0}$, if required.

Even within this specific context, it is in principle possible to generalize in many different ways. For that reason, it is important to be able to test quantitatively some of the consequences of local scale-invariance. Here the calculation of the two-time response function $R(t, s)$ is one of the easiest tasks and has the advantage that prediction are relatively easy to test in simulations, for example by considering an integrated response such as the thermoremanent magnetization $\mathrm{M}_{\mathrm{TRM}}(t, s)$.

The central idea of LSI is the decomposition of the stochastic Langevin equation into a deterministic part which can possess local scale-invariance and into a noise part which does break this. Remarkably, the noise part is usually built in such a way that any $n$-point function of the full theory can be reduced exactly to a certain $m$-point function of the noiseless theory (where $m \geq n$ ). This reduction depends on the Bargman superselection rules which are proven only for $z=2$ at the time of writing. Then the symmetries of the noiseless part yield conditions on the form of the $m$-point functions. In certain cases, such as the two-time response functions, these constraints are enough to fix the functional form, see eq. (34).

In models where the deterministic part of the Langevin equation is linear, LSI can be proven, for example in the spherical model [103], the $1 D$ Glauber-Ising model $[103,65]$, bosonic particle-reaction models [9] or certain simple growth models $[110$, 12]. Furthermore, there is a systematic way to construct LSI-invariant non-linear equations $[115,9]$. Since these are not identical to the Langevin equations one usually 
starts from [71], numerical evidence for those systems is crucial, in the absence of exact solutions.

In order to be able to carry out meaningful tests, one must first show that simple ageing (on which LSI is built) holds true. For non-conserved ferromagnets this is thought to be certain [20] and we have discussed the evidence that this remains so for disordered, non-glassy Ising models. In most numerically studied systems, only $M_{\mathrm{TRM}}(t, s)$ has been compared with LSI. Provided that corrections to scaling are treated carefully, in all cases the results are consistent with LSI, see tables 2 and 3. The fact that $M_{\mathrm{TRM}}(t, s)$ as measured in a noisy system is consistent with the integral of (34) found from the symmetries of the noiseless theory, even if $z \neq 2$, is a strong indication that an extension of the Bargman superselection rules to $z \neq 2$ should exist.

At present, the only other observables measured have been space-time responses and autocorrelations in the two- and three-dimensional Ising models[58, 61] and in the two-dimensional Potts- $q$ models $(q=2,3,8)$ [89]. It would be important to consider different quantities, e.g. alternating susceptibilities.

When turning to critical spin glasses, although there is evidence that simple ageing applies, the agreement of the data with LSI was only partial. It should be recalled that the analysis performed relied on a rapid change between quasi-static and scaling behaviour, which might be an oversimplification. Remarkably, in the presence of disorder we have seen evidence that the non-equilibrium exponents may depend on the distribution of the random exchange couplings $J_{i, j}$, independently on whether the system was glassy or not. Since this is a highly controversial issue, further tests would be most welcome.

At present, the available evidence ${ }^{11}$ from both analytical solved models and numerical studies supports the predictions of local scale-invariance for the two-time response (and in some cases also correlation) functions. This suggests that the kind of Langevin equation used in the description of these models should possess a fundamental, hitherto unsuspected and non-trivial dynamical symmetry underlying certain stochastic Langevin equations. Already the present examples show that the calculation of time-dependent quantities such as the thermoremanent magnetization is considerably simplified and leads to results not available as yet by other methods. Local scale-invariance, similar in spirit to the tremendously successful techniques of conformal field-theory, has the potential of leading to a much more profound understanding of non-equilibrium critical phenomena.

Finally, comparison of simulational results with experimental evidence for both glassy and non-glassy systems (see tables $5 \& 7$ ) points to some systematic differences which need to be clarified.

Acknowledgements : We thank Wolfhard Janke for his invitation to write this review, F. Baumann, I.A. Campbell, S.B. Dutta, T. Enss, A. Gambassi, A. Röthlein and J. Unterberger for fruitful collaborations on the thématique reviewed here and acknowledge the support by the Deutsche Forschungsgemeinschaft through grant no. PL 323/2. This work was also supported by the franco-german binational programme PROCOPE and by CINES Montpellier (projet pmn2095).

${ }^{11}$ Of course models such as the celebrated zero-range process, see [42, 50], which lacks a proper spatial structure should be excluded from this kind of consideration. 


\section{References}

1. S. Abriet and D. Karevski, Eur. Phys. J. B37, 47 (2004).

2. S. Abriet and D. Karevski, Eur. Phys. J. B41, 79 (2004).

3. S.M. Allen and J.W. Cahn, Acta Metall. 27, 1085 (1979).

4. J.-O. Andersson, T. Jonsson and J. Mattsson, Phys. Rev. B54, 9912 (1996).

5. A. Andreanov and A. Lefèvre, Europhys. Lett. 76, 919 (2006).

6. A. Annibale and P. Sollich, J. Phys. A39, 2853 (2006).

7. V. Bargman, Ann. of Math. 56, 1 (1954).

8. F. Baumann, M. Henkel, M. Pleimling and J. Richert, J. Phys. A: Math. Gen. 38 6623, (2005).

9. F. Baumann, S. Stoimenov and M. Henkel, J. Phys. A39, 4095 (2006).

10. F. Baumann and M. Pleimling, J. Phys. A39, 1981 (2006).

11. F. Baumann and A. Gambassi, J. Stat. Mech. at the press cond-mat/0610260.

12. F. Baumann and M. Henkel, J. Stat. Mech. at the press cond-mat/0611652.

13. F. Baumann, S.B. Dutta and M. Henkel, in preparation.

14. A.A. Belavin, A.M. Polyakov and A.B. Zamolodchikov, Nucl. Phys. B241, 333 (1984).

15. L. W. Bernardi and I. A. Campbell, Phys. Rev. B56, 5271 (1997).

16. L. Berthier, J.L. Barrat and J. Kurchan, Eur. Phys. J. B11, 635 (1999).

17. L. Berthier, P.C.W. Holdsworth and M. Sellitto, J. Phys. A34, 1805 (2001).

18. L. Berthier and J.-P. Bouchaud, Phys. Rev. B66, 054404 (2002).

19. J.-P. Bouchaud, V. Dupuis, J. Hammann and E. Vincent, Phys. Rev. B65, 024439 (2001).

20. A.J. Bray, Adv. Phys. 43, 357 (1994).

21. A.J. Bray and A.D. Rutenberg, Phys. Rev. E49, R27 (1994); E51, 5499 (1995).

22. P. Calabrese and A. Gambassi, Phys. Rev. E65, 066120 (2002).

23. P. Calabrese and A. Gambassi, Phys. Rev. E66, 066101 (2002).

24. P. Calabrese and A. Gambassi, Phys. Rev. B66, 212407 (2002).

25. P. Calabrese and A. Gambassi, J. Phys. A38, R181 (2005).

26. P. Calabrese, A. Gambassi and F. Krzakala, J. Stat Mech. Theor. Exp. P06016 (2006).

27. S.A. Cannas, D.A. Stariolo and F.A. Tamarit, Physica A294, 362 (2001).

28. J.L. Cardy in E. Brézin and J. Zinn-Justin (eds), Fields, strings and critical phenomena, Les Houches XLIX, North Holland (Amsterdam 1990).

29. M.E. Cates and M.R. Evans (eds) Soft and fragile matter, IOP Press (Bristol 2000).

30. C. Chatelain, J. Phys. A36, 10739 (2003).

31. E.V. Colla, L.K. Chao, M.B. Weissman and D.D. Viehland, Phys. Rev. Lett. 85, 3033 (2000).

32. E.V. Colla, L.K. Chao and M.B. Weissman, Phys. Rev. B63, 134107 (2001).

33. A. Crisanti and F. Ritort, J. Phys. A36, R181 (2003)

34. L.F. Cugliandolo, in Slow Relaxation and non equilibrium dynamics in condensed matter, Les Houches Session 77 July 2002, J-L Barrat, J Dalibard, J Kurchan, M V Feigel'man eds (Springer, 2003); cond-mat/0210312.

35. L.F. Cugliandolo, J. Kurchan, and G. Parisi, J. Physique I4, 1641 (1994).

36. C. de Dominicis and L. Peliti, Phys. Rev. B18, 353 (1978).

37. V. Dupuis, thèse de doctorat (Paris XI Orsay, 2002).

38. V. Dupuis, E. Vincent, M. Alba and J. Hammann, Eur. Phys. J. B29, 19 (2002). 
39. V. Dupuis, E. Vincent, J.-P. Bouchaud, J. Hammann, A. Ito and H.A. Katori, Phys. Rev. B64, 174204 (2001).

40. V. Dupuis, F. Bert, J.-P. Bouchaud, J. Hammann, F. Ladieu, D. Parker and E. Vincent, cond-mat/0406672.

41. T. Enss, M. Henkel, A. Picone and U. Schollwöck, J. Phys. A37, 10479 (2004).

42. M.R. Evans and T. Hanney, J. Phys. A38, R195 (2005).

43. A.A. Fedorenko and S. Trimper, Europhys. Lett. 74, 89 (2006).

44. D.S. Fisher and D.A. Huse, Phys. Rev. B38, 373 (1988).

45. P. di Francesco, P. Mathieu et D. Sénéchal, Conformal field theory, Springer (Heidelberg 1997).

46. A. Gambassi, J. Phys. Conf. Series 40, 13 (2006).

47. C. Godrèche and J.M. Luck, J. Phys. A33, 1151 (2000).

48. C. Godrèche and J.-M. Luck, J. Phys. A33, 9141 (2000).

49. C. Godrèche and J.M. Luck, J. Phys. Cond. Matt. 14, 1589 (2002).

50. C. Godrèche, in [64] (cond-mat/0604276).

51. C.R. Hagen, Phys. Rev. D5, 377 (1972).

52. M. Henkel, J. Stat. Phys. 75, 1023 (1994).

53. M. Henkel, Conformal invariance and critical phenomena, Springer (Heidelbeg 1999).

54. M. Henkel, M. Pleimling, C. Godrèche and J.-M. Luck, Phys. Rev. Lett. 87, 265701 (2001)

55. M. Henkel, Nucl. Phys. B641, 405 (2002).

56. M. Henkel, M. Paessens and M. Pleimling, Europhys. Lett. 62, 644 (2003)

57. M. Henkel and J. Unterberger, Nucl. Phys. B660, 407 (2003).

58. M. Henkel and M. Pleimling, Phys. Rev. E68, 065101(R) (2003).

59. M. Henkel and G.M. Schütz, J. Phys. A37, 591 (2004).

60. M. Henkel, M. Paessens and M. Pleimling, Phys. Rev. E69, 056109 (2004).

61. M. Henkel, A. Picone and M. Pleimling, Europhys. Lett. 68, 191 (2004).

62. M. Henkel and M. Pleimling, J. Phys. Cond. Matt. 17, S1899 (2005).

63. M. Henkel and M. Pleimling, Europhys. Lett. 69, 524 (2005).

64. M. Henkel, M. Pleimling and R. Sanctuary (eds), Ageing and the glass transition, Springer Lecture Notes in Physics, Springer (Heidelberg 2007).

65. M. Henkel, T. Enss and M. Pleimling, J. Phys. A39, L589 (2006).

66. M. Henkel and M. Pleimling, Europhys. Lett. 76, 561 (2006).

67. M. Henkel, J. Phys. Cond. Matt. at the press (2007), cond-mat/0609672.

68. M. Henkel and M. Pleimling, in preparation.

69. C.L. Henley, Phys. Rev. Lett. 54, 2030 (1985).

70. H. Hinrichsen, J. Stat. Mech. L06001 (2006).

71. P. Hohenberg and B.I. Halperin, Rev. Mod. Phys. 49, 435 (1977).

72. D.A. Huse and C.L. Henley, Phys. Rev. Lett. 54, 2708 (1985)

73. D.A. Huse, Phys. Rev. B40, 304 (1989).

74. H. Ikeda, Y. Endoh and S. Itoh, Phys. Rev. Lett. 64, 1266 (1990).

75. W. Janke, in [64].

76. H.K. Janssen, B. Schaub and B. Schmittmann, Z. Phys. B73, 539 (1989).

77. H.K. Janssen, in G. Györgyi et al. (eds) From Phase transitions to Chaos, World Scientific (Singapour 1992), p. 68

78. P.E. Jönsson, H. Yoshino, P. Nordblad, H. Agura Katori and A. Ito, Phys. Rev. Lett. 88, 257204 (2002).

79. T. Jörg, Phys. Rev. B 73, 224431 (2006). 
80. H.A. Kastrup, Nucl. Phys. B7, 545 (1968).

81. H. G. Katzgraber, M. Körner, and A. P. Young, Phys. Rev. B 73, 224432 (2006).

82. N. Kawashima and H. Rieger, in Frustrated magnetic systems, H. Diep (ed.) (World Scientific, 2004).

83. J. Kurchan, Phys. Rev. E66, 017101 (2002).

84. Z.W. Lai, G.F. Mazenko and O.T. Valls, Phys. Rwev. B37, 9481 (1988).

85. R.L. Leheny and S.R. Nagel, Phys. Rev. B57, 5154 (1998).

86. V. Likodimos, M. Labardi and M. Allegrini, Phys. Rev. B61, 14440 (2000).

87. V. Likodimos, M. Labardi, X.K. Orlik, L. Pardi and M. Allegrini, Phys. Rev. B63, 064104 (2001).

88. E. Lippiello and M. Zanetti, Phys. Rev. E61, 3369 (2000).

89. E. Lorenz and W. Janke, Europhys. Lett. at the press (2007).

90. P. Mayer, PhD thesis, King's college London (2004).

91. P. Mayer, S. Léonard, L. Berthier, J.P. Garrahan and P. Sollich, Phys. Rev. Lett. 96, 030602 (2006).

92. G.F. Mazenko, Phys. Rev. E69, 016114 (2004).

93. O. Narayan and A.P. Young, Phys. Rev. E64, 021104 (2001)

94. T.J. Newman and A.J. Bray, J. Phys. A23, 4491 (1990).

95. U. Niederer, Helv. Phys. Acta 45, 802 (1972).

96. G. Ódor, J. Stat. Mech. L11002 (2006).

97. R. Paul, S. Puri and H. Rieger, Europhys. Lett. 68, 881 (2004).

98. R. Paul, S. Puri and H. Rieger, Phys. Rev. E71, 061109 (2005).

99. R. Paul, G. Schehr and H. Rieger, cond-mat/0611246.

100. M. Perroud, Helv. Phys. Acta 50, 233 (1977).

101. M. Picco, F. Ricci-Tersenghi and F. Ritort, Eur. Phys. J. B21, 211 (2001).

102. A. Picone and M. Henkel, J. Phys. A35, 5575 (2002).

103. A. Picone and M. Henkel, Nucl. Phys. B688, 217 (2004).

104. M. Pleimling and A. Gambassi, Phys. Rev. B71, 180401(R) (2005).

105. M. Pleimling and I.A. Campbell, Phys. Rev. B72, 184429 (2005).

106. J.J. Ramasco, M. Henkel, M.A. Santos and C.A. de Silva Santos, J. Phys. A37, 10497 (2004).

107. R. Rammal and A. Benoît, Phys. Rev. Lett. 55, 649 (1985).

108. M. Rheinstädter, K. Knorr and H. Rieger, Phys. Rev. B69, 144427 (2004).

109. G.F. Rodriguez, G.G. Kenning and R. Orbach, Phys. Rev. Lett. 91, 037203 (2003).

110. A. Röthlein, F. Baumann and M. Pleimlimg, Phys. Rev. E at the press cond-mat/0609707.

111. G. Schehr and R. Paul, Phys. Rev. E72, 016105 (2005).

112. G. Schehr and R. Paul, J. Phys. Conf. Series 40, 27 (2006).

113. D.K. Shenoy, J.V. Selinger, K.A. Grüneberg, J. Naciri and R. Shashidhar, Phys. Rev. Lett. 82, 1716 (1999).

114. A.G. Schins, A.F.M. Arts and H.W. de Wijn, Phys. Rev. Lett. 70, 2340 (1993).

115. S. Stoimenov and M. Henkel, Nucl. Phys. B723, 205 (2005).

116. L.C.E. Struik, Physical ageing in amorphous polymers and other materials, Elsevier (Amsterdam 1978).

117. I.S. Suzuki and M. Suzuki, Phys. Rev. B68, 094424 (2003).

118. N.G. van Kampen, Stochastic processes in physics and chemistry, $2^{\text {nd }}$ edition, North Holland (Amsterdam 1992). 
119. E. Vincent, V. Dupuis, M. Alba, J. Hammann and J.-P. Bouchaud, Europhys. Lett. 50, 674 (2000).

120. E. Vincent, in [64].

121. H. Yoshino, K. Hukusghima and H. Takayama, Phys. Rev. B66, 064431 (2002).

122. W. Zippold, R. Kühn and H. Horner, Eur. Phys. J. B13, 531 (2000).

123. R. Zwanzig, Nonequilibrium statistical mechanics, Oxford University Press (Oxford 2001). 